\title{
Scalar Dark Matter candidates
}

\author{
C. Bøhm \\ Denys Wilkinson Laboratory, 1 Keble Road, OX1 3RH, Oxford, England, UK \\ P. Fayet \\ Laboratoire de Physique Théorique de l'ENS, 24 rue Lhomond, \\ 75231 Paris Cedex 05, France ${ }^{1}$
}

\begin{abstract}
We investigate the possibility that Dark Matter could be made of scalar candidates and focus, in particular, on the unusual mass range between a few MeV's and a few GeV's. After showing why the Lee-Weinberg limit (which usually forbids a Dark Matter mass below a few GeV's) does not necessarily apply in the case of scalar particles, we discuss how light candidates $\left(m_{d m}<O(\mathrm{GeV})\right)$ can satisfy both the gamma ray and relic density constraints. We find two possibilities. Either Dark Matter is coupled to heavy fermions (but if $m_{d m} \lesssim 100 \mathrm{MeV}$, an asymmetry between the Dark Matter particle and antiparticle number densities is likely to be required), or Dark Matter is coupled to a new light gauge boson $U$. The (collisional) damping of light candidates is, in some circumstances, large enough to be mentioned, but in most cases too small to generate a non linear matter power spectrum at the present epoch that differs significantly from the Cold Dark Matter spectrum. On the other hand, heavier scalar Dark Matter particles (i.e. with $m_{d m} \gtrsim O(\mathrm{GeV})$ ) turn out to be much less constrained. We finally discuss a theoretical framework for scalar candidates, inspired from theories with $N=2$ extended supersymmetry and/or extra space dimensions, in which the Dark Matter stability results from a new discrete (or continuous) symmetry.
\end{abstract}

\section{Introduction}

The nature of Dark Matter (DM) is still a challenging question in cosmology. While ordinary matter appears to be a very unlikely explanation [1-3] to a set of well-known observations, the solution based on the existence of a new kind

$\overline{1 \text { UMR }}$ 8549, Unité mixte du CNRS et de l'Ecole Normale Supérieure. 
of neutral, stable and Weakly Interacting Massive Particles (WIMPs) [4] is still under investigations. The preferred theoretical framework which supports such particles, namely supersymmetry, remains hypothetical and possible discrepancies between observations and some of the Cold Dark Matter theoretical predictions are still under debate [5-7]. This makes worthwhile to explore less conventional possibilities, as we shall do here.

To satisfy the relic density requirement ${ }^{2}$ as well as other experimental constraints, the Dark Matter mass (and, in particular, the mass of the presently favored candidate, the lightest neutralino), is generally thought to lie between $m_{d m} \sim O(\mathrm{GeV})$ (in fact $O(30 \mathrm{GeV}$ ) for neutralinos, assuming unification constraints) and $O(\mathrm{TeV})[8,9]$. While this range certainly appears natural and promising, surprisingly enough, "light" annihilating particles with a mass below a few GeV's and no significant direct coupling to the $Z$ boson do not appear experimentally excluded as yet, as we shall see here. This immediately raises the question of their relic abundance.

Annihilating Dark Matter particles with a mass below a few GeV's are generally expected to be ruled out because they would overclose the Universe [10]. But this argument, based on a relationship between the cosmological parameter $\Omega_{d m} h^{2}$ and the Dark Matter annihilation cross section $\sigma_{a n n}$, applies only to fermionic candidates for which $\sigma_{a n n}$ is seen to be proportional to $m_{d m}^{2}$. In the case of scalar particles, however, the situation is different. The estimated relic abundance of such candidates may in fact vary considerably, depending on if and how these are taken to interact with ordinary matter. For scalar candidates interacting through Higgs exchanges [11] - which interact very weakly with ordinary matter - the resulting weakness of the annihilation cross sections tends to lead to an excessively large relic abundance, especially in the case of light scalar particles. We shall discuss here other situations, in which the scalar candidate interacts with ordinary matter either through Yukawa exchanges of new fermions (such as mirror fermions), or through the exchanges of a new neutral gauge boson. The annihilation cross sections can then be largely independent of the Dark Matter mass or depend on two low masses (namely the Dark Matter mass and the mass of the exchanged particles), so the relationship between $\sigma_{a n n}$ and $\Omega_{d m} h^{2}$ eventually turns out to (mostly) constrain the mass and coupling constants of the exchanged particles. We show that in such situations scalar particle masses below a few GeV's are indeed possible, as far as the relic density argument is concerned.

At this stage it gets necessary to perform a further analysis. Indeed even if they have the correct relic density light scalar candidates could still be ruled out by other astrophysical constraints such as, for instance, the precise measurement of gamma ray fluxes below a few GeV's. By comparing the observed fluxes with

$\overline{2 \Omega_{\mathrm{dm}} h^{2}} \sim 0.1$, where $H=100 h \mathrm{~km} \mathrm{~s}^{-1} \mathrm{Mpc}^{-1}$. 
those expected from Dark Matter residual annihilations into ordinary particles, it comes out that particles lighter than a few hundred MeV's are excluded if their annihilation cross section times velocity $\left(\sigma_{\text {ann }} v_{r e l}\right)$ behaves mostly as a constant [12] (unless there is an asymmetry between the Dark Matter and anti Dark Matter number densities). On the other hand, such particles could escape past and possibly present gamma ray detection experiments if $\sigma_{\text {ann }} v_{r e l}$ was significantly dominated, at the freeze-out epoch, by a term proportional to the square of the Dark Matter velocity, i.e. if $\sigma_{\text {ann }} v_{r e l} \approx b v_{d m}^{2}$ at the freeze-out epoch (with $b$ a constant).

The aim of this paper is therefore to check whether or not scalar Dark Matter candidates can have properties which are compatible, simultaneously, with

- the relic density requirement,

- the measured gamma ray fluxes,

- and, finally, the present experimental limits from particle physics experiments.

After giving the generic Feynman rules which enter our calculations, we discuss, in Section 3, how theories with a new light gauge boson $U$ may be constructed, the general properties of $U$ interactions, as well as possible implications for Higgs bosons. We compute, in Section 4, the annihilation cross section of light scalar candidates into fermion-antifermion pairs. We then look for the conditions that allow this cross section (times relative velocity) to give rise to a satisfactory relic density and be significantly dominated by a term proportional to $v_{d m}^{2}$ at the freeze-out epoch (which is of interest for $m_{d m} \lesssim 100 \mathrm{MeV}$ ). Because the possible existence of non-chiral Yukawa couplings for scalar particles appears very important regarding the relic density issue, we also rederive for comparison the annihilation cross section of fermionic candidates in Section 5. Section 6 is dedicated to the question of the collisional damping generated by light scalar candidates. To that respect, we compute the elastic scattering cross section of Dark Matter with neutrinos and determine their associated damping scale. We then discuss, in Section 7, the case of an asymmetry between the number densities of Dark Matter particles and antiparticles, as a way to evade the gamma ray constraint on $m_{d m} \lesssim 100 \mathrm{MeV}$. Finally, we investigate in Section 8 a theoretical framework (inspired from $N=2$ extended supersymmetry and/or theories with extra space dimensions) in which a light "spin-0 gauge boson" (e.g. a "spin-0 photon") could turn out to be a viable Dark Matter candidate.

\section{Dark Matter couplings}

Although the Dark Matter stability could result from the extreme smallness of its couplings to ordinary particles, we find more elegant to impose a new symmetry, which may be either discrete or continuous. 
The case of self conjugate Dark Matter particles $\left(\mathrm{dm} \equiv \mathrm{dm}^{*}\right)$ will be described by introducing a new $Z_{2}$ discrete symmetry (called $M$-parity and denoted $M_{p}$ ), while the case $\mathrm{dm} \neq \mathrm{dm} *$ will be obtained by imposing other discrete or continuous symmetries (e.g. $U(1)$ ), that we call $M$ symmetry and denote simply by $M$ (to contrast with $M_{p}$, dedicated to the $Z_{2}$ symmetry only).

In the case of a $M$-parity, somewhat similar to $R$-parity in supersymmetric theories [13], we can distinguish between " $M$-even" and " $M$-odd" particles (their associated fields transforming according to $\phi \stackrel{M_{p}}{\longrightarrow}-\phi$ ). The denomination is slightly different in the case of a $M$ symmetry, for which we shall distinguish between particles transforming trivially or non-trivially under the $M$ symmetry. $\mathrm{t}$ If the LMP (i.e. the lightest of the $M$-odd particles, or the lightest of the particles transforming non trivially under the new symmetry) is neutral and uncolored, then we end up with a possible Dark Matter candidate. This is actually what we shall assume from now on.

\subsection{Direct Yukawa couplings of scalar Dark Matter to matter particles}

Similarly to neutralinos in supersymmetry, we may expect Dark Matter particles to be coupled to ordinary fermions $(f)$ and other particles $(F)$. These $F$ particles denote new fermions if Dark Matter is bosonic or new bosons if Dark Matter is fermionic. The new states $F$ and Dark Matter particles transform in the same way under the new symmetry, so we can introduce non-diagonal couplings of the type $\mathrm{dm}-\bar{f}-F$ which are expected to be invariant under $M$-parity or $M$ symmetry, whatever the Dark Matter spin (that we shall restrict to be 0 or $1 / 2$ for simplicity). We shall set the masses of charged $F$ 's to be typically above $\sim 100 \mathrm{GeV}$ 's so as to be compatible with unfruitful searches for new charged particles.

Let us now express the corresponding relevant Feynman rules in the case of scalar Dark Matter. The $F$ 's denote new spin-1/2 fermions and the Yukawa couplings $\mathrm{dm}-\bar{f}-F$ can be written as

$$
\delta\left(C_{l} \overline{f_{L}} F_{R}+C_{r} \overline{f_{R}} F_{L}\right)+\text { h.c. } .
$$

This leads to the following Feynman rule:

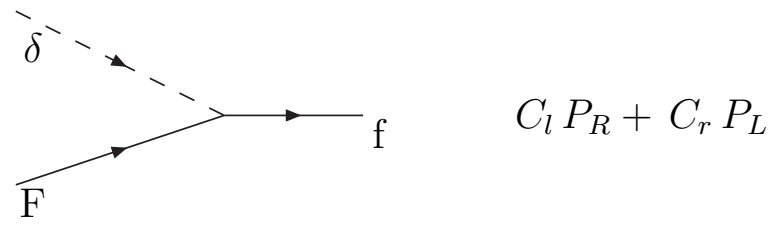

where $P_{L}$ and $P_{R}$ denote the two chiral projectors. 
Here $\delta$ stands for the spin-0 Dark Matter field, and $C_{l}$ and $C_{r}$ denote the strengths of its Yukawa couplings to the left-handed and right-handed ordinary fermion fields $f_{L}$ and $f_{R}$, respectively. The left-handed fermion fields $f_{L}$ transform as members of electroweak doublets and their right-handed counterparts as singlets, so in the most relevant case for which Dark Matter is an electroweak singlet (possibly mixed with the neutral component of an electroweak triplet, as we shall discuss later), the new fermion fields $F_{R}$ should transform as members of electroweak doublets and their left-handed counterparts $F_{L}$ as singlets. In other words, the new fermion fields $F$ appear to be mirror partners of the ordinary fermion fields $f$.

As also discussed later in Section 8, one can indeed construct models (possibly but not necessarily inspired from extended supersymmetry and/or extra space dimensions), in which quarks and leptons (built from left-handed electroweak doublets and right-handed singlets), are supplemented by mirror partners (built from right-handed doublets and left-handed singlets). Ordinary as well as mirror quarks and leptons acquire their masses, as usual, after spontaneous gauge symmetry breaking through their couplings to one or several electroweak Englert-Brout-Higgs doublets. Experimental constraints require mirror charged fermions to be sufficiently heavy, more than about $100 \mathrm{GeV}$ 's at least, which may be easily realized as soon as their Yukawa couplings to the Higgs are taken to be sufficiently large. In addition, mirror neutrinos, which did not show up in $Z$ decays, should be heavier than about $40 \mathrm{GeV}$ 's at least. This may be realized, for example, by considering them (at least in a first approximation) as 4-component Dirac fermions, with large Dirac mass terms connecting right-handed mirror neutrino fields (members of electroweak doublets), with associated left-handed extra singlet ("sterile") neutrino fields (without introducing large "Majorana mass terms" for the latter).

Furthermore, a "mirror-parity" symmetry distinguishing between the ordinary and mirror sectors prevents ordinary fermions to mix with those of the mirror sector. Nevertheless, ordinary and mirror fermions may still be coupled together, in a gauge-invariant way. The corresponding Yukawa couplings would then involve "mirror-odd" scalars - e.g., in the simplest case, a neutral electroweak singlet, for which one can immediately write a gauge-invariant mass term. The lightest of these scalars, i.e. in fact the "LMP", would then be stable thanks to $M$-parity (as soon as it is lighter than all mirror fermions), providing, in this way, a possible Dark Matter candidate. This particle would interact with ordinary matter, or annihilate in pairs, through mirror fermion exchanges with ordinary matter fermions. This constitutes, precisely, one of the main subjects of the paper. 


\subsection{For comparison, direct Yukawa couplings of spin-1/2 Dark Matter parti- cles.}

Let us now also express, for the purpose of future comparisons, the corresponding Feynman rules in the usual case of spin-1/2 Dark Matter particles. The $F$ 's then represent new boson fields. We make the assumption that they are scalar fields (e.g. the squarks and sleptons, in the case of supersymmetry), for simplicity. Their non-diagonal Yukawa couplings to Dark Matter and ordinary fermion fields $f$ may in principle involve, similarly to the coupling $\tilde{f}-f-\chi^{0}$ in supersymmetry, the two quantities $\left[\begin{array}{lll}C_{l} & F \overline{f_{L}} \delta_{R}+h . c .\end{array}\right]$ and

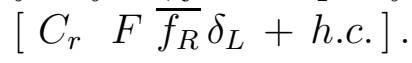

- If Dark Matter is a Majorana particle (so that $\delta_{R}=\left(\delta_{L}\right)^{c}$ ), and in the simplest case for which the $F$ and $\delta_{R}$ fields would appear as eigenstates of the weak isospin $T_{3}$, the electroweak gauge invariance requires that one or the other of the two Yukawa couplings $C_{l}$ and $C_{r}$ must vanish identically, i.e. that the couplings of a Majorana Dark Matter particle with a spin-0 field $F$ be chiral, i.e. for example

$$
C_{l} F \overline{f_{L}} \delta_{R}+\text { h.c. , }
$$

which gives rise to the rule:

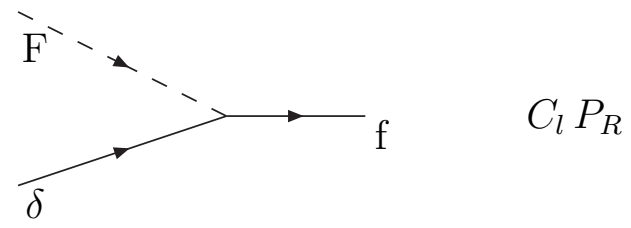

- If Dark Matter is a Majorana particle, but $\delta$ (or $F$ ) appear as mixings of field components with different values of $T_{3}$, then one expects that nonchiral couplings such as for instance

$$
C_{l} F \overline{f_{L}} \delta_{R}+C_{r} F \overline{f_{R}} \delta_{L}+\text { h.c. },
$$

be generated, in a way compatible with the (spontaneously broken) $S U(2) \times$ $U(1)$ gauge symmetry. (Such a situation may occur, for example, in supersymmetric theories, where $F$ would denote a new state resulting from the mixing of the two spin- 0 sfermion fields $\widetilde{f_{L}}$ and $\widetilde{f_{R}}$, and $\delta$ a Majorana neutralino.) We then get the following Feynman rule:

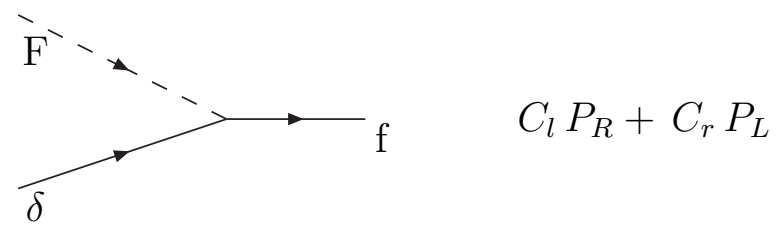


- If, on the other hand, Dark Matter is a Dirac particle (so that $\delta_{L}$ and $\delta_{R}$ denote independent field components), then the Yukawa couplings of the spin-0 fields $F$ to Dark Matter and ordinary fermions may also be written in a non-chiral way as:

$$
C_{l} F \overline{f_{L}} \delta_{R}+C_{r} F \overline{f_{R}} \delta_{L}+\text { h.c. . }
$$

This again gives rise to:

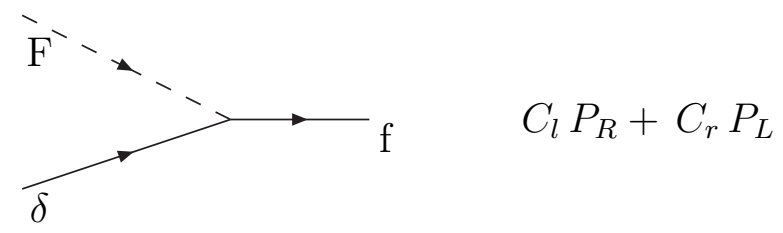

\subsection{Couplings to $Z$ or Higgs bosons}

Dark Matter particles may interact with ordinary matter through $Z$ boson exchanges, as in the case of a neutralino, or scalar neutrino. They should then necessarily be heavy, otherwise they would have been pair-produced in $Z$ decays. A light dark matter candidate should have no significant direct coupling to the $Z$ boson, but it could still interact with ordinary matter through the exchanges of other spin-1 gauge bosons (as we shall discuss in the next subsection), or of spin-0 Higgs bosons.

In the case of scalar Dark Matter particles interacting with matter through spin-0 Higgs boson exchanges, as discussed for example in the simple model of Ref. [11], the weakness of the Higgs couplings to ordinary fermions leads, in general, to a too small annihilation cross section and therefore a too large relic abundance of dark matter particles, unless one is ready to compensate for that by considering a large dark matter/Higgs boson coupling (which would tend to make the theory non-perturbative), or to assume that Dark Matter is close to in mass to $m_{\mathrm{Higgs}} / 2$ - and therefore relatively heavy. Since Higgs or $Z$ exchanges do not really favor light Dark Matter particles, we shall disregard this possibility in what follows.

\subsection{Couplings to a new gauge boson}

Dark Matter particles can also interact with ordinary fermions $f$ through couplings to a new neutral gauge boson $U$, should it exist (in fact a $U$ boson has already been proposed earlier but in another context $[14,15]$ ). We shall 
denote the coupling constant(s) between two Dark Matter particles and the $U$ boson (i.e. the $U$-charge of the Dark Matter field) by $C_{U}$ in the case of a scalar Dark Matter field, by $C_{U_{r}}$ in the case of a Majorana Dark Matter field (rewritten as a right-handed fermion field) and by $C_{U_{l}}$ and $C_{U_{r}}$ in the case of a Dirac Dark Matter field, while the couplings of the $U$ boson with ordinary chiral fermion fields will be denoted by $f_{U_{l}}$ and $f_{U_{r}}$, respectively. We then end up with the following rules:

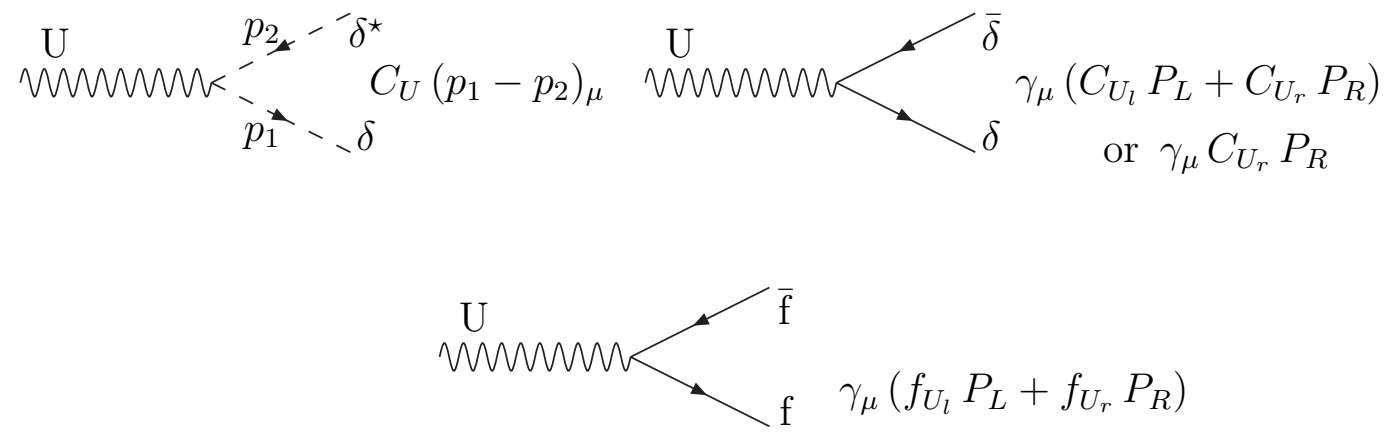

\section{More on theories with a spontaneously broken extra $U(1)$ gauge symmetry}

\subsection{An extra $U(1)$, and its spontaneous breaking}

We shall work within the framework of spontaneously broken gauge theories, in which the Standard Model gauge group is extended so as to include, at least, an extra $U(1)$ factor. The new resulting neutral gauge boson, $U$, acquires a mass from the spontaneous breaking of the $S U(3) \times S U(2) \times U(1) \times$ extra $U(1)$ gauge symmetry into the $S U(3) \times U(1)$ subgroup of QCD $\times \mathrm{QED}^{3}$. This breaking, and the subsequent mass of the $U$ boson, may be obtained explicitly from the non-vanishing vacuum expectation values of two electroweak Higgs doublets $\varphi_{i}$, such as those present in supersymmetric theories [13]; or of two Higgs doublets + an extra singlet (that we may call here $\sigma$ ), allowing us to increase, if necessary, the scale $F$ at which the extra $U(1)$ symmetry is spontaneously broken, so that the effects of the exchanges, or direct production, of the new neutral gauge boson $U$ may be sufficiently small $[14,15]$. Or simply (in the absence of supersymmetry) by the v.e.v.'s of only one electroweak Higgs doublet $(\varphi)$ and one extra singlet $(\sigma)[16]$. Such additional singlets interact-

3 While this extra $U(1)$ was originally introduced in connection with spontaneous supersymmetry breaking, this initial motivation has faded away and it should now be considered, independently of supersymmetry. 
ing with the Higgs doublets $\varphi$ may be naturally present in many situations, including in particular non-minimal versions of the supersymmetric standard model [13,17], supersymmetric grand-unified theories, theories with extended supersymmetry and/or extra dimensions, .... In contrast with the Higgs doublet(s) $\varphi$, the extra Higgs singlet(s) $\sigma$ can never couple directly to ordinary quarks and leptons, a point which will be of importance later. A general analysis discussing in particular which are the possible extra $U(1)$ symmetries which may be gauged ${ }^{4}$ (with gauge coupling $g$ "), and how the associated current $J$ " may mix with the $S U(2) \times U(1)$ current $J_{3}-\sin ^{2} \theta J_{e m}$ to give the new current $J_{U}$ and the weak neutral current $J_{Z}$, and many possible effects of a new light gauge boson $U$, is given in Ref. [18].

\subsection{Possible effects on neutral current phenomenology: towards a light $U$ bo- son}

Clearly the question of possible $Z-U$ mixing effects, which might spoil the (now very well-known) properties of the $Z$ boson and corresponding neutral current $J_{Z}$, is of primary importance, and we must make sure that such effects be sufficiently small, in the situations we are interested in. Such a mixing may indeed be absent - as, for example, in the simplest situations considered in $[13,14]$, when the two Higgs doublets $\varphi_{i}$ are taken to acquire equal v.e.v.'s (corresponding to $\tan \beta=1$ in modern susy language, or $1 / x=1$ in the axion language).

But if we then recover exactly the usual properties and neutral current interactions of the $Z$ boson, we still have to worry about the effects of $U$-boson exchanges on weak neutral current processes, which have to be sufficiently small. In a number of situations, in which the extra $U(1)$ symmetry would be broken at or around the electroweak scale $v$, for example by two Higgs doublets, these $U$-exchange amplitudes would be proportional to $g^{\prime \prime 2} / m_{U}^{2}$ i.e. precisely to $G_{F}$, just as for the standard $Z$-exchange weak amplitudes, which turned out to be in contradiction with experimental results on neutral current processes [14].

This led us (apart from the obvious possible solution to increase the mass of the $U$ so as to make it very heavy) to pay special attention to situations in which the extra $U(1)$ gauge coupling constant $g$ " is taken to be small, together with the mass $m_{U}$ of the new neutral gauge boson $U$; propagator effects then become essential when estimating $U$ exchange amplitudes. This is usually

4 We also assume that the anomalies associated with the extra $U(1)$ gauge symmetry are suitably cancelled, which may be easily realized, e.g. by using the $B-L$ and $Y$ symmetry generators in the fermionic sector, or by introducing mirror fermions, .... 
(at least for a symmetry breaking scale $F \gtrsim$ electroweak scale) sufficient to make the $U$-exchange amplitudes (proportional to $g^{\prime \prime 2} /\left(m_{U}^{2}-q^{2}\right)$ ) negligible compared to the $Z$-exchange amplitudes (proportional to $\left(g^{2}+g^{\prime 2}\right) / m_{Z}^{2}$, i.e. to $G_{F}$ ), in high-energy scattering experiments (with $\left|q^{2}\right| \gg m_{U}^{2}{ }^{5}$ ) [14] - a first success against too large unwanted effects of the new gauge boson!

In the case of experiments performed at lower energies and therefore smaller values of the momentum transfer $\left|q^{2}\right| \lesssim m_{U}^{2}$, on the other hand, $U$ boson exchanges could still contribute quite significantly or even excessively to neutrino scattering cross sections. Extra scattering amplitudes of neutrinos on electrons, fixed by the ratios $f_{U \nu} f_{U e} /\left(m_{U}^{2}-q^{2}\right)$ (denoting by $f_{U \nu}$ and $f_{U e}$ the $U$-charges of the neutrino and electron fields, respectively), should be added to the usual standard model weak-interaction scattering amplitudes, which appear with a coefficient $2 G_{F} \sqrt{2}$. The neutrino-electron scattering cross section has been measured at low energy $(\approx 20-50 \mathrm{MeV}$, corresponding to small momentum transfer of a few MeV's), by the LAMPF and LSND experiments, which found no significant deviation from the Standard Model [19]. The resulting constraint may be written as $\left|f_{U \nu} f_{U e}\right| /\left(m_{U}^{2}-q^{2}\right) \lesssim G_{F}$ or simply $\left|f_{U \nu} f_{U e}\right| / m_{U}^{2} \lesssim G_{F}$ if the $U$ is heavier than a few MeV's. This may lead us, subsequently, to consider very small values of the $U$ coupling to the neutrinos (or, possibly, a very small coupling to the electron).

\subsection{A very light $U$ boson does not decouple, even in the limit of vanishing gauge coupling!}

Once we take this direction of a small $g$ " i.e. of a rather weakly coupled and light $U$ boson, we might naïvely think that it is sufficient to consider a sufficiently small $g$ " so as to get rid of all the unwanted effects of the new gauge boson $U$. The interactions of this new boson, however, should not be taken as negligible, even in the limit in which the extra $U(1)$ gauge coupling constant g" gets extremely small! Indeed an equivalence theorem shows that, in this low mass and low coupling limit (i.e. for small $m_{U} \approx g " F$ and small $g$ ", their fixed ratio defining the scale $F$ at which the extra $U(1)$ symmetry gets spontaneously broken ${ }^{6}$ ), the longitudinal polarisation state of the massive spin-1 $U$ boson does not decouple! It behaves, instead, very much as the massless spin-0 Goldstone boson associated with the spontaneous breaking of the extra $U(1)$, now considered as a global symmetry [14,15]. This is quite similar to

$\overline{5}$ The $\left|q^{2}\right|$ term then remains essential it the expression of the $U$ boson progator, while it may be neglected compared to $m_{Z}^{2}$, in the corresponding $Z$ boson propagator.

${ }^{6}$ This scale $F$ is determined by the v.e.v.'s of the various Higgs doublets and singlets ... responsible for the spontaneous breaking of the extra $U(1)$ symmetry. 
the equivalence theorem of supersymmetry, according to which a light spin$3 / 2$ gravitino does not decouple in the limit $\kappa=\sqrt{8 \pi G_{N}} \rightarrow 0$, but interacts (proportionally to $\kappa / m_{3 / 2}$ i.e. to $1 / d \approx 1 / \Lambda_{s s}^{2}$ ) very much like the massless spin-1/2 goldstino of spontaneously broken global supersymmetry [20].

We should therefore pay a particular attention to these residual interactions of the longitudinal polarisation state of the $U$ boson, which behaves very much as a (quasi massless) spin-0 Goldstone boson. In fact it could appear as quasimassless axionlike particle, which might show up in $\psi, \Upsilon$ and (if lighter than $\simeq 1 \mathrm{MeV}$ ) positronium decays $[14,15,18,21]$ ! However, if we look at things more closely, the phenomenology of such models varies considerably, depending on whether the current to which the new gauge boson $U$ couples is purely vectorial (as e.g. in the one-Higgs-doublet + one-Higgs-singlet situation detailed in Ref. [16]), or has also an axial part, as it occurs in general when there is more than one Higgs doublet, as in supersymmetric theories (cf. the general discussion in Ref. [18]). In these latter cases a very light $U$ boson behaves very much as a spin- 0 axion (in contrast with the simpler case of a purely vectorial $U$ current). And we then run the risk of having this particle excluded experimentally, very much as it happened for a standard axion.

\subsection{The "invisible U-boson" mechanism}

Fortunately enough a $U$ boson, however, can be made "invisible" (in particle physics experiments) if the corresponding extra $U(1)$ symmetry breaking scale $F$ is taken to be sufficiently large (as compared to the electroweak scale $v \simeq$ $246 \mathrm{GeV}$ ) by using, for example, a large Higgs singlet vacuum expectation value $(\langle\sigma\rangle)$ responsible for the generation of this large symmetry breaking scale $(F \approx\langle\sigma\rangle \gg v)[14,15]$. (The same mechanism, incidentally, can also be used to make the interactions of the spin- 0 axion in particle physics almost "invisible", in correspondence with what happens for the spin-1 $U$ boson, in agreement with the equivalence theorem mentioned earlier ${ }^{7}$.) If we denote

$\sigma=\frac{h-i a}{\sqrt{2}}$ this extra Higgs field transforming non-trivially under the extra $U(1)$ symmetry, the longitudinal degree of freedom of the very light spin-1 $U$ boson behaves very much as the corresponding - also almost "invisible" (eaten) pseudoscalar Goldstone boson. The latter, almost identical to $a$ in the large $F$ limit, is then very weakly coupled to ordinary matter.

\subsection{A purely vectorial $U$ current}

The neutral current $J_{U}$ to which the $U$ boson couples is obtained from the

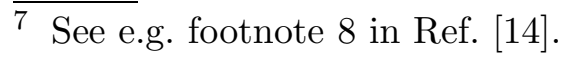


extra $U(1)$ current $J$ ", after taking into account an additional contribution proportional to $J_{3}-\sin ^{2} \theta J_{e m}$ arising from $Z-U$ mixing effects, when such effects are present $[18,22]$. It involves in general an axial part (if there is more than one Higgs doublet) as well as a vector part which turns out to involve (as far as leptons and quarks are concerned) a linear combination of the $B, L$ and electromagnetic currents.

However there are also simpler situations in which the extra $U(1)$ current turns out to be purely vectorial[16]. Indeed, to avoid any potential difficulty with an axionlike behavior of the $U$ boson (and having to resort to an extra $U(1)$ symmetry broken "at a high scale" $F$ significantly larger than the electroweak scale), we shall often concentrate for simplicity on such situations. Then no (potentially troublesome) axionlike behavior of the longitudinal polarisation state of the $U$ boson (associated with non-vanishing axial couplings to quarks and leptons) will manifest, and the extra $U(1)$ symmetry is in this case not necessarily systematically constrained to be broken at a scale $F$ higher than the electroweak scale. Explicit examples have been detailed, with the spontaneous gauge symmetry breaking triggered by the standard model Higgs doublet $\varphi$, supplemented with an electroweak singlet $\sigma$ which only transforms under the extra $U(1)$ symmetry, and does not couple directly to quarks and leptons [16]. After mixing effects between neutral gauge bosons are taken into account, the $Z$ boson field and $Z$ current expressions remain essentially unchanged when one considers, as we do here, sufficiently small values of the extra $U(1)$ gauge coupling $g$ ". The quark and lepton contribution to the $U$ current turns out to be a linear combination of the corresponding $B, L$ and electromagnetic currents $[16,18]$.

\subsection{Implications for the Higgs sector}

Within the above class of models the simplest situation is obtained when the standard model Higgs doublet $\varphi$ transforms only under the electroweak $S U(2) \times U(1)$ symmetry (and is alone responsible for the $W^{ \pm}$and $Z$ masses), and the new singlet Higgs field $\sigma$ only under the extra $U(1)$ symmetry (and is alone responsible for the $U$ mass), so that no $Z-U$ mixing effect occurs at this level. The quark and lepton contribution to the $U$ current appears simply as a linear combination of the corresponding $B$ and $L$ currents (such as, for example, $B-L)$. The imaginary part $(a)$ of the additional Higgs singlet $\sigma$ generates the extra ("longitudinal") degree of freedom of the massive $U$ boson. Its real part $(h)$ corresponds to an additional neutral scalar (" $C P$ even") physical Higgs field, which does not couple directly to quarks and leptons.

The latter singlet field ( $h$, given from $\sigma \rightarrow \frac{h+w}{\sqrt{2}}$ ) may (in general) or may not 
mix with the usual standard model Higgs field ( $H$, given from $\left.\varphi \rightarrow \frac{H+v}{\sqrt{2}}\right)$, depending on the existence and magnitude of the dimensionless coupling of the $|\varphi|^{2}|\sigma|^{2}$ gauge invariant quartic interaction in the scalar potential, which may be present in such models (as already in [17]), leading after translation of the Higgs fields to a $(H+v)^{2}(h+w)^{2}$ interaction term. In the absence of such a term that would be responsible for doublet-singlet mixing effects, the usual phenomenology of the standard model Higgs boson $(H)$ remains essentially unchanged. The new singlet Higgs boson $(h)$, which has a largely arbitrary mass and no direct coupling to quarks and leptons, is expected to decay instead into $U U$ pairs through a $h U U$ coupling, leading to invisible as well as visible decay modes - depending on the invisible ( $\mathrm{dm} \mathrm{dm}$ or $\nu \bar{\nu})$ or visible (into $e^{+} e^{-}, \ldots$, depending on $m_{U}$ ) decay modes of the $U$ boson.

In general, however, a mixing between the $H$ (doublet) and $h$ (singlet) real scalar fields is expected to occur, as indicated above. As a result of this $H-$ $h$ mixing, the single Higgs boson of the standard model $H$ would then get replaced by a pair ${ }^{8}$ of Higgs mass eigenstates $H_{1,2}$. Each of them would be coupled to quarks and leptons proportionally to their mass $\left(m_{q}\right.$ and $\left.m_{l}\right)$, as in the standard model, times the cosine, or sine, of the mixing angle $(\xi)$ between the two doublet and singlet components (originating from the real parts of the $\varphi$ and $\sigma$ fields, respectively). The corresponding decay rates of such Higgs bosons $H_{i}$ into standard model particles would then be obtained as e.g. $\Gamma_{S M}\left(m_{H_{2}}\right) \cos ^{2} \xi$ and $\Gamma_{S M}\left(m_{H_{1}}\right) \sin ^{2} \xi$, in terms of the standard model decay rates for a Higgs boson of the corresponding mass. This would of course significantly affect the experimental searches for Higgs boson signals. Given the possible hints for the direct observation of a Higgs signal at LEP [23], one could even contemplate the possibility of having one of these Higgs mass eigenstates arond $116 \mathrm{GeV}$ 's, for example, and the other at a different mass.

New possible decay modes of these Higgs mass eigenstates $H_{i}$ into $U U$ pairs, for example, would also have to be considered in this case. They would lead to new Higgs decays into e.g. $e^{+} e^{-} e^{+} e^{-}, e^{+} e^{-}+$missing energy, and totally invisible decay modes from $U \rightarrow \nu \bar{\nu}$ or $\mathrm{dm}$ dm pairs. In addition, the quartic $h h U U$ coupling would also lead to further cascade modes following the decay of the heavier Higgs boson into the lighter one, according to $H_{2} \rightarrow H_{1} U U$.

\subsection{Back to Dark Matter candidates}

Being reassured on the possibility of reproducing, in various possible ways, the successful phenomenology of the Standard Model, with possibly but not necessarily interesting new features in the Higgs sector, associated with doublet-

8 Or possibly more, in more elaborate situations. A similar phenomenon may also occur in non-minimal supersymmetric extensions of the standard model. 
singlet mixing and new Higgs decay modes, we can now proceed and concentrate again on our Dark Matter candidates.

As mentioned earlier the field associated with it could be, in the simplest case, a neutral electroweak singlet but, more generally, it may also appear as the result of a mixing between the neutral components of singlets, triplets and doublets of the electroweak gauge group. However a significant contribution from electroweak doublets is usually strongly disfavored, as it would generally induce an unwanted diagonal coupling to the $Z$ boson, yielding a too large contribution to the invisible decay width of the $Z$. Furthermore, charged partners under the electroweak symmetry of non-singlet Dark Matter particles should also be heavy, i.e. typically above $\sim 100 \mathrm{GeV}$ 's.

\section{Annihilation cross sections of scalar candidates}

We can now compute the annihilation cross sections of a pair of Dark Matter scalar particles into a pair fermion/antifermion (the expressions of the squared matrix amplitudes are given explicitly in the Appendix). We disregard the annihilations into photons which, because they involve radiative processes, are generally expected to be smaller than the annihilations into fermion pairs.

We shall see that, in some circumstances, the annihilation cross sections of spin-0 particles do not depend significantly on the mass of the incoming particles, say in our case the Dark Matter mass. Instead, they depend on other parameters, namely the masses and couplings of the exchanged particles. This characteristic is in fact of crucial importance since it allows to evade the lower limit of $\sim 2$ GeV's given by Lee and Weinberg for spin-1/2 leptons on the basis of Fermi interactions (a similar limit, $O(\mathrm{GeV}$ ), is found also in the framework of weak interactions and fermionic candidates).

\subsection{Compatibility with relic density}

The annihilation cross sections of scalar candidates into ordinary fermions have already been worked out within a supersymmetric framework for sneutrinos [24] but we shall rederive their expressions because the mass of the outcoming particles (i.e. ordinary fermions) was neglected, and the terms proportional

to $v_{d m}^{2}$ were disregarded when the $v_{d m}$-independent contributions (or, almost equivalently, the S-wave terms) were found not to be identically null. Note that, to avoid radiative processes that would involve both the fermion $F$ and the $U$ boson, we shall consider that Dark Matter cannot be coupled to $F$ and $U$ simultaneously. This allows us to derive their contributions independently. 
F exchanges

We start by the coupling $\operatorname{dm}-f-F$, assuming for simplicity that the Yukawa couplings $C_{l}$ and $C_{r}$ are real. We want to check that the cross sections associated with Dark Matter candidates lighter than a few GeV's and coupled to fermions $F$ can satisfy, for the mass range we are interested in, the simple relationship imposed by relic density calculations: $\Omega_{d m} h^{2} \sim O\left(10^{-27}-10^{-28}\right)$ $\mathrm{cm}^{3} \mathrm{~s}^{-1} /\left\langle\sigma_{\text {ann }} v_{\text {rel }}\right\rangle$, or equivalently $\left\langle\sigma_{\text {ann }} v_{\text {rel }}\right\rangle \sim O\left(10^{-26}-10^{-27} \mathrm{~cm}^{3} \mathrm{~s}^{-1}\right)$ with $\Omega_{d m} h^{2} \sim 0.1$, as determined by several experiments [25,26]. (Actually the required cross section is expected to be two times smaller in the case of self-conjugate Dark Matter particles, as compared to non-self-conjugate ones.) Such values of the cross sections may be obtained, in particular, if the annihilation cross section $\sigma_{a n n}$ is mostly independent of the Dark Matter mass, as we shall see.

When $\mathrm{dm} \neq \mathrm{dm}^{\star}$, the annihilation cross section associated with the process $\mathrm{dm} \mathrm{dm}^{\star} \rightarrow f \bar{f}$ (which proceeds via a single " $t$ " channel, see Appendix 9.1.4) is given, in the local limit approximation $\left(m_{F} \gg m_{d m} \geq m_{f}\right)$, by:

$$
\sigma_{a n n} v_{r e l} \simeq \frac{1}{4 \pi} \sqrt{1-\frac{m_{f}^{2}}{m_{d m}^{2}}}\left(1-\frac{m_{f}^{2}}{m_{d m}^{2}}+v_{d m}^{2}\right) C_{l}^{2} C_{r}^{2} / m_{F}^{2}
$$

We quote here only the dominant terms but the full expression can be found in the Appendix. For $\mathrm{dm}=\mathrm{dm}^{\star}$ (i.e. self-conjugate Dark Matter particles, see Appendix 9.1.3), one gets essentially the same expression but with a larger numerical coefficient:

$$
\sigma_{a n n} v_{r e l} \simeq \frac{1}{\pi} \sqrt{1-\frac{m_{f}^{2}}{m_{d m}^{2}}}\left(1-\frac{m_{f}^{2}}{m_{d m}^{2}}+v_{d m}^{2}\right) C_{l}^{2} C_{r}^{2} / m_{F}^{2}
$$

The factor four in between (1) and (2) originates from the fact that one has to take into account the $t$ and $u$ channels plus their interference terms (all of them contributing with the same weight). Although there is no P-wave term in the case of self-conjugate Dark Matter particles, there exists a $v_{d m}^{2}$ contribution that originates from the development of the S-wave term at the second order.

Our results indicate that $\sigma_{a n n} v_{r e l}$ is almost independent of the Dark Matter mass under the condition of non chiral couplings. As a result, one can obtain $\sigma_{\text {ann }} v_{r e l} \sim O\left(10^{-26}\right)-O\left(10^{-27}\right) \mathrm{cm}^{3} \mathrm{~s}^{-1}$, almost independently of the Dark Matter mass, simply by adjusting the quantity $C_{l} C_{r} / m_{F}$. This actually illustrates a situation in which relic density calculations appear inefficient to rule out certain values of the Dark Matter mass. 
As an example, $\Omega_{d m} h^{2} \sim 0.1$ can be achieved with $C_{l} C_{r} \sim 0.01-0.1$ and $m_{F} \simeq O(100 \mathrm{GeV})-O(\mathrm{TeV})$ for any value of the Dark Matter mass below a few GeV's. In fact, a more refined approach would consist in using a Boltzmann code [27] to determine carefully the value of $\left\langle\sigma_{a n n} v_{r e l}\right\rangle$ (and therefore $m_{F}$ and $C_{l} C_{r}$ etc. ) depending on the freeze-out epoch ${ }^{9}$. But we think that there is no need to go to this level of accuracy for light particles, especially since, as we shall see (and this is one of the main points of this paper), relic density calculations do not provide the most stringent constraint on candidates lighter than one hundred MeV's.

In the case of chiral couplings $C_{l} C_{r}=0$, the above expressions (1) and (2) of the annihilation cross sections become meaningless ${ }^{10}$. One must return to less simplified expressions of the squared matrix amplitudes (see Appendix 9.1.3, 9.1.4) which show that the cross sections of candidates lighter than a few GeV's (and with chiral couplings) remain significantly too small to allow for an acceptable relic density (unless one increases the couplings $C_{l}$ or $C_{r}$ up to values which seem very unlikely and problematic).

We therefore conclude, at this stage, that scalar candidates with $m_{d m} \lesssim$ $O(\mathrm{GeV})$, coupled to heavy fermions $F$, can fulfill the relic density requirement only if they have non chiral couplings.

But such a condition could turn out to eventually exclude the possibility of light Dark Matter candidates. Indeed, the contribution of a charged $F$ to the muon and electron anomalous magnetic moments, in the case of non chiral couplings, is given by $\delta a_{\mu, e}^{m_{F} \gg m_{\mu, e}} \simeq \frac{C_{l} C_{r} m_{\mu, e}}{16 \pi^{2} m_{F}}$. Here $\frac{C_{l} C_{r}}{m_{F}}$ denotes either $\left(\frac{C_{l} C_{r}}{m_{F}}\right)_{\mu}$ or $\left(\frac{C_{l} C_{r}}{m_{F}}\right)_{e}$, depending on whether one estimates the contribution to the muon or electron $g-2$, respectively. If not cancelled, $\delta a_{\mu}^{m_{F}}$ has to be lower than a few times $10^{-9}$ (extendable up to $510^{-9}$ ) and $\delta a_{e}^{m_{F}}$ lower than a few times $10^{-11}$ (up to $810^{-11}$ ), to be compatible with the differences between the experimental values and the Standard Model predictions. Since the quan-

9 The estimate of the freeze-out epoch also depends on the annihilation cross section and on the Dark Matter mass but through a logarithm: $m_{d m} / T_{F O} \sim$ $17+\ln \left(\left\langle\sigma_{a n n} v_{r e l}\right\rangle / 10^{-26} \mathrm{~cm}^{3} \mathrm{~s}^{-1}\right)+\ln \left(m_{d m} / \mathrm{GeV}\right)+\ln \sqrt{m_{d m} / T_{F O}}$. We then expect the errors on the estimate of $m_{d m} / T_{F O}$ not to affect significantly our conclusions. Also it is important to note that the relation between the cosmological parameter and the annihilation cross section depends linearly on $m_{d m} / T_{F O}$, see [28] for details.

${ }^{10}$ When $m_{d m}$ is very close but not equal to $m_{\mu}$, annihilations into muons proceed through a velocity-dependent cross section. This actually has no effect on relic density calculations if Dark Matter is coupled to muons as it is coupled to electrons, but this could, perhaps, be important for the estimate of gamma ray fluxes from Dark Matter particles slightly heavier than the muon since photons would be generated mostly through electron-positron pairs. 
tity $\frac{C_{l} \quad C_{r}}{m_{F}}$ also enters the expressions of the corresponding annihilation cross sections into $\mu^{+} \mu^{-}$or $e^{+} e^{-}$pairs, it is possible to rewrite the cross section as

$$
\begin{aligned}
\sigma_{a n n} v_{r e l} & \simeq \frac{1}{(4) \pi} \sqrt{1-\frac{m_{\mu, e}^{2}}{m_{d m}^{2}}}\left(1-\frac{m_{\mu, e}^{2}}{m_{d m}^{2}}+v_{d m}^{2}\right)\left(\frac{16 \pi^{2} \delta a_{\mu, e}^{m_{F}} \gg m_{\mu, e}}{m_{\mu, e}}\right)^{2} \\
& \lesssim O\left(10^{-29}\right) \mathrm{cm}^{3} \mathrm{~s}^{-1}
\end{aligned}
$$

(if $\delta a_{e}<10^{-11}$ and $\delta a_{\mu}<10^{-9}$ ), which is much lower than the value $\sigma_{a n n} v_{r e l} \sim O\left(10^{-26}-10^{-27} \mathrm{~cm}^{3} \mathrm{~s}^{-1}\right)$ required for an acceptable relic abundance. It is worth noting however that $\sigma_{a n n} v_{r e l}$ can reach $610^{-28}$ or $610^{-29} \mathrm{~cm}^{3} \mathrm{~s}^{-1}$ (for non self-conjugate particles) with the maximum values of $\delta a_{e}$ and $\delta a_{\mu}$ and potentially up to $10^{-26}-10^{-27} \mathrm{~cm}^{3} \mathrm{~s}^{-1}$ if one sums over all the annihilation channels.

Thus, excepted in marginal situations, light Dark Matter particles coupled to fermions $F$ and with a mass $m_{d m}<O(\mathrm{GeV})$ can get the proper relic density only if $a_{\mu, e}^{m_{F}}$ is cancelled by another contribution. This may nevertheless happen if, in addition to the charged $F$, there also exists for instance charged scalars $\left(H^{-}\right)$and neutral fermions $\left(F^{0}\right)$, cf. Appendix 9.4. In this case indeed, significantly larger values of the annihilation cross section would be allowed, but at the price of a fine tuning. In fact such a cancellation may also occur by introducing a new (pseudo)scalar particle. For instance, in the framework of theories originating from $N=2$ extended supersymmetry or extra dimensions ( $c f$. Section 8), the Dark Matter candidate is supplemented by another neutral spin-0 particle, which may also be relatively light. Both the Dark Matter and the other spin-0 particle would have non-chiral couplings to fermion/mirror fermion pairs but one would be a scalar while the other would be a pseudoscalar. Each separate contribution to the muon or electron $g-2$ is expected to be too large, but their sum would be, on the other hand, naturally small. Large values of the annihilation cross section would then be allowed, without being in conflict with $g-2$ constraints. In all these cases, the particle spectrum required to get an acceptable relic abundance should be within the reach of future LHC experiments.

\section{$U$ boson}

Let us now estimate the efficiency of the Dark Matter annihilation process through the $s$-channel production of a gauge boson $U$. We assume that the direct Dark Matter coupling to the $Z$ is null (or extremely small). As mentioned previously, the coupling between the $U$ boson and ordinary fermions $(U-f-\bar{f})$ can be written as $\gamma^{\mu}\left(f_{U_{l}} P_{L}+f_{U_{r}} P_{R}\right)$ and the coupling between 
the $U$ and the Dark Matter particles as $C_{U}$. The corresponding annihilation cross section is then given by

$$
\sigma_{a n n} v_{r e l} \simeq v_{d m}^{2} \sqrt{1-\frac{m_{f}^{2}}{m_{d m}^{2}}} \frac{C_{U}^{2}\left[4 m_{d m}^{2}\left(f_{U_{l}}^{2}+f_{U_{r}}^{2}\right)-m_{f}^{2}\left(f_{U_{l}}^{2}-6 f_{U_{l}} f_{U_{r}}+f_{U_{r}}^{2}\right)\right]}{12 \pi\left(m_{U}^{2}-4 m_{d m}^{2}\right)^{2}}
$$

(we assume $m_{U}>2 m_{d m}$ ). The interesting feature here is that the $\mathrm{S}$-wave term is naturally suppressed (as noted in [24] for sneutrinos), due to the derivative nature of the couplings between the $U$ boson and the Dark Matter particles.

If the $U$ boson is very heavy (more than a few hundred GeV's) and the Dark Matter mass lower than a few GeV's, then one expects the cross section given in eq. (3) to be too small to allow for an acceptable relic abundance. On the contrary, if the $U$ boson turns out to be light (less than a few GeV's), one may obtain the appropriate value of the annihilation cross section provided the couplings $C_{U}$ and $f_{U_{l}}, f_{U_{r}}$ be sufficiently small. This is actually fortunate since the smallness of the coupling $U-f-\bar{f}$ is also needed to fulfill, in particular, experimental limits from the muon and electron's anomalous magnetic moments (see Appendix 9.4). Basically, $f_{U_{l}}$ and $f_{U_{r}}$ associated with either $U-\mu-\bar{\mu}$ or $U-e-\bar{e}$ should be smaller than about $310^{-6}\left(\frac{m_{U}}{\mathrm{MeV}}\right)\left(\frac{\delta a_{\mu}}{10^{-9}}\right)^{1 / 2}$ (in fact $310^{-4}\left(\frac{\delta a_{\mu}}{10^{-9}}\right)^{1 / 2}$ if $\left.m_{U}<m_{\mu}\right)$ or $710^{-5}\left(\frac{m_{U}}{\mathrm{MeV}}\right)\left(\frac{\delta a_{e}}{10^{-11}}\right)^{1 / 2}$ (assuming $f_{U_{l}}=f_{U_{r}}$ and depending whether $m_{U}$ is greater than $m_{\mu}$ or not), in order to be compatible with both the muon and electron's $g-2$.

Taken at face value, the couplings $f_{U_{l}}$ (and $f_{U_{r}}$ ) can give rise to an annihilation cross section (into a pair muon-antimuon or electron-positron depending on the Dark Matter mass) of the order of $10^{-26}-10^{-27} \mathrm{~cm}^{3} \mathrm{~s}^{-1}$ provided that the $U$ boson happens to be more strongly coupled to the Dark Matter than to ordinary fermions. As an illustration, to obtain $\Omega_{d m} h^{2} \sim 0.1$ with, for instance, $f_{U_{l}} \sim f_{U_{r}} \sim 410^{-4}\left(\delta a_{e} \simeq 310^{-12}\right), m_{d m} \sim 4 \mathrm{MeV}$ and $m_{U} \sim 10$ $\mathrm{MeV}, C_{U}$ should be of the order of $\sim(2.5-8) 10^{-3}$. More generally, one obtains the correct relic density if $C_{U} f_{U_{l}} \sim(3-12) 10^{-8}\left(\frac{m_{U}}{\mathrm{MeV}}\right)^{2}\left(\frac{m_{d m}}{\mathrm{MeV}}\right)^{-1}$.

In fact, two expressions of $C_{U}$ are displayed in Table 1, depending on whether $\mathrm{dm} \mathrm{dm} \rightarrow e^{+} e^{-}$or $\mathrm{dm} \mathrm{dm} \rightarrow \mu^{+} \mu^{-}$is the dominant channel. If $\left\langle\sigma_{a n n} v_{r e l}\right\rangle_{d m d m \rightarrow \mu^{+} \mu^{-}}=\left\langle\sigma_{a n n} v_{r e l}\right\rangle_{d m d m \rightarrow e^{+} e^{-}}$, then the product $\left(f_{U_{l}} C_{U}\right)_{\mu}$ should be equal to $\left(f_{U_{l}} C_{U}\right)_{e}$. Since $C_{U}$ is related to Dark Matter only, one gets $\left(f_{U_{l}}\right)_{\mu}=\left(f_{U_{l}}\right)_{e}$ (which can be further translated into a relationship between $\delta a_{e}$ and $\left.\delta a_{\mu}\right)$.

In conclusion, scalar Dark Matter particles can be significantly lighter than a few GeV's (thus evading the generalisation of the Lee-Weinberg limit for weakly-interacting neutral fermions) if they are coupled to a new (light) gauge boson or to new heavy fermions $F$ (through non chiral couplings and poten- 
tially provided one introduces additional particles). Let us now determine if they can be compatible with the observed production of gamma rays.

\subsection{Compatibility with gamma rays}

In [12], it was shown that if light Dark Matter candidates $\left(m_{d m}<O(100) \mathrm{MeV}\right)$ have a roughly "constant" annihilation cross section of $\left\langle\sigma_{a n n} v_{r e l}\right\rangle \sim O\left(10^{-26}\right.$ $10^{-27} \mathrm{~cm}^{3} \mathrm{~s}^{-1}$ ) (as needed to satisfy the relic density requirement), they would yield too much gamma rays as compared with present observations ${ }^{11}$. They would be allowed by COMPTEL, OSSE and EGRET data [29], on the other hand, if their cross section (times the relative velocity) at the moment of the residual annihilations was $\sim 10^{-31}\left(10^{-29}\right.$ or $\left.10^{-28}\right) \mathrm{cm}^{3} \mathrm{~s}^{-1}$ for $m_{d m} \sim$ 1 (10 or 100) MeV respectively. This actually suggests that light Dark Matter particles with a constant cross section are ruled out while particles that would mainly annihilate through a velocity-dependent cross section would be allowed. Thereby, this requires that the Dark Matter velocity-independent cross section be at least $\sim 10^{5}$ (resp. $10^{3}$ or $10^{2}$ ) times smaller than the velocity-dependent cross section estimated at the freeze-out epoch $\left(t_{F O}\right)$, for $m_{d m} \sim 1$ ( 10 or 100) $\mathrm{MeV}$ (and $\left\langle\sigma_{\text {ann }} v_{\text {rel }}\right\rangle \sim 10^{-26} \mathrm{~cm}^{3} \mathrm{~s}^{-1}$ ). Let us now check if the cross sections displayed above have the proper characteristics.

\section{Residual annihilations through $F$ exchanges}

We have seen previously that the coupling $\mathrm{dm}-\bar{f}-F$ should be non chiral in order to get the correct relic density. However, in this case, the S-wave term in the cross section appears of the same order of magnitude than the velocitydependent term. Therefore light scalar candidates (with $m_{d m} \lesssim O(100) \mathrm{MeV}$ ) that would get the proper relic density, are likely to be excluded because they would yield a too large gamma ray production (unless one invokes an asymmetry between the Dark Matter particle and antiparticle number densities since, in this case, no significant residual annihilations are expected). Only searches for new particles in accelerator experiments would then constrain the properties of charged F's (and of the additional spectrum needed to cancel its associated $g-2$ contributions).

\footnotetext{
${ }^{11}$ We assume here that there is no asymmetry between the Dark Matter particle and antiparticle number densities so that one can use residual annihilations in galaxy clusters or centres of galaxies as a constraint on the properties of light Dark Matter particles.
} 
The gamma ray constraint appears much easier to satisfy with a $U$ boson because the cross section is naturally "S-wave" suppressed (the constant term vanishes identically). The value of the annihilation cross section inside the galactic centre is expected to be suppressed by the square of the Dark Matter velocity. We therefore expect the cross section to be roughly $310^{-6}$ times what is needed at the freeze-out epoch, leading to a radiative flux $\phi \sim 210^{-5}$ $\left(\frac{m_{d m}}{\mathrm{MeV}}\right)^{-2} \mathrm{~cm}^{-2} \mathrm{~s}^{-1}$ for $m_{d m} \in O(1-100) \mathrm{MeV}$ (using a NFW profile [30]). This flux would be smaller with another Dark Matter halo profile. As a result, $O(1-100) \mathrm{MeV}$ particles could indeed have escaped previous gamma ray experiments, although masses $m_{d m} \sim O(\mathrm{MeV})$ seem (under the condition of a NFW profile) very close to experimental limits.

To summarize, the production of gamma rays provides a much stronger constraint on scalar particles with $m_{d m} \lesssim 100 \mathrm{MeV}$ and on the nature of their interactions, than relic density calculations. This study indeed indicates that i) annihilations through $F$ exchanges are forbidden unless there exists an asymmetry between the Dark Matter and anti Dark Matter number densities and ii) a gamma ray signature from the galactic centre at low energy could be due to the existence of a light new gauge boson. The range beyond a few hundred MeV's, on the other hand, is less constrained by gamma ray fluxes (whether Dark Matter is coupled to a $U$ boson or a $F$ particle) but some constraints on the production of $D+{ }^{3} H_{e}$ [31] may also require a velocity-dependent cross section.

Let us mention a point concerning the existence of the $U$ boson that might be important especially since WMAP's result [32]. Because the annihilation cross section appears to be S-wave suppressed, the residual annihilations of light Dark Matter are expected to be important when the Dark Matter velocity and number density are the highest. We would therefore expect a "gamma ray" signature in the centres of galaxies or clusters of galaxies at low redshift but there might also exist a signature at larger redshift where the (mean) Dark Matter number density is supposed to be bigger (due to redshift effects). This signature, should it exist, could be a kind of early reionization, or perhaps an indirect source of reionization. Its importance would depend on the Dark Matter velocity and number density that is embedded in structures formed at a given redshift. Although we did not perform any calculations, it is worth mentioning this effect because it might allow to constrain the scenario of light Dark Matter particles coupled to a $U$ boson. 


\section{Annihilation cross sections of spin $\frac{1}{2}$ candidates}

Although this is not the purpose of our paper, we now return for comparison to a more usual situation where Dark Matter is made of spin- $\frac{1}{2}$ particles. Due to a Lee-Weinberg-type limit for "weakly-interacting" massive leptons, we expect fermionic candidates with a mass smaller than a few GeV's to be ruled out. If coupled to a scalar $F$, then the annihilation cross section of spin-1/2 Dark Matter particles is expected to be proportional to $m_{f}^{2} / m_{F}^{4}$ or $m_{d m}^{2} / m_{F}^{4}$. This is actually much too small to prevent them from overclosing the Universe (unless one considers the exchange of a relatively light neutral scalar $F$ that would not be coupled to the $Z$ boson).

Calculations displayed in the literature have shown that for Dirac fermions both the $\mathrm{S}$ and $\mathrm{P}$-wave cross sections are proportional ${ }^{12}$ to $m_{d m}^{2} / m_{F}^{4}$ (assuming that the exchanged particle, namely a scalar $F$, is much heavier than the Dark Matter and quoting only the most relevant terms) while for Majorana fermions, the S-wave term is proportional to $m_{f}^{2} / m_{F}^{4}$ and the $\mathrm{P}$-wave term to $m_{d m}^{2} / m_{F}^{4}$, unless the couplings are non-chiral (i.e $C_{l} C_{r} \neq 0$ ) [33]. In this case, indeed, it is known that the S-wave annihilation cross section of Majorana particles is proportional to $m_{d m}^{2} / m_{F}^{4}$ instead ${ }^{13}$ of $m_{f}^{2} / m_{F}^{4}$.

Since spin-1/2 Majorana Dark Matter particles have the following annihilation cross section (keeping only the dominant terms):

$$
\begin{array}{r}
\sigma_{a n n} v_{r e l} \sim \frac{1}{32 \pi m_{F}{ }^{4}} \sqrt{1-\frac{m_{f}^{2}}{m_{d m}^{2}}}\left\{\left(2 C_{l} C_{r} m_{d m}+\left(C_{l}^{2}+C_{r}^{2}\right) m_{f}\right)^{2}\right. \\
\left.+\frac{4 v_{d m}^{2}}{3}\left(m_{d m}^{2}\left(2 C_{l}^{4}+9 C_{l}^{2} C_{r}^{2}+2 C_{r}^{4}\right)\right)+\ldots\right\},
\end{array}
$$

one should keep the S-wave term in a situation where the couplings are non chiral. This is expected to be important for Dark Matter particles much heavier

${ }^{12}$ Keeping the dominant terms, the annihilation cross section of spin- $1 / 2$ Dirac fermions is given by:

$$
\sigma_{a n n} v_{r e l} \sim \frac{1}{32 \pi m_{F}^{4}} \sqrt{1-\frac{m_{f}^{2}}{m_{d m}^{2}}}\left(C_{l}^{2}+C_{r}^{2}\right)^{2} m_{d m}^{2}\left\{1+\frac{7 v_{d m}^{2}}{3}\right\} .
$$

${ }^{13}$ For instance, in supersymmetry, non chiral couplings as the result of a mixing angle between the two stop fields $\widetilde{t_{L}}$ and $\widetilde{t_{R}}$, have to be taken into account in the estimate of the annihilation cross section of neutralinos into $t \bar{t}$ (through a stop exchange). 
than either the tau (or the bottom quark, but slightly lighter than the top, i.e. $\left.m_{\tau, b} \ll m_{d m} \lesssim m_{t}\right)$, or heavier than the top $\left(m_{d m}>m_{t}\right)$. In these cases, indeed, the gamma ray flux - proportional to the Dark Matter residual annihilation cross section - is expected to be larger than previous estimates based on a cross section proportional to $m_{d m}^{2} v_{d m}^{2} / m_{F}^{4}$ or $m_{f}^{2} / m_{F}^{4}$.

More generally, the S and P-wave terms that we find by assuming non chiral couplings appear, at the freeze-out epoch, somewhat larger than the P-wave term estimated in the presence of chiral couplings of similar magnitude. This suggests that the lower bound on the Dark Matter mass obtained in the case of non chiral couplings is somewhat smaller than the bound derived when the couplings are chiral. However, despite this characteristic, the lower limit on the Dark Matter mass is still expected to be of a few GeV's (assuming $m_{F}>m_{W}$, which could perhaps be contested if the primordial annihilations mainly proceed into neutrinos through neutral $F$ exchanges). Therefore, the mass range below a few GeV's is very likely to require scalar Dark Matter particles (rather than spin-1/2 particles), assuming Dark Matter has indeed to annihilate to get the proper relic density.

\section{Collisional damping effect}

Dark Matter is generally thought to interact weakly enough to be considered as collisionless regarding structure formation [4]. However, a more detailed study shows that even Dark Matter particles with weak interactions yield damping effects in the linear matter power spectrum [34,35] at a given scale. The issue is then to determine whether this scale is of cosmological interest or not.

The answer to that question is related to the quantity $\sigma_{e l} /\left(m_{d m} / \mathrm{GeV}\right)$, denoting the ratio of the Dark Matter elastic scattering cross section to its mass (measured in $\mathrm{GeV}$ ). If this ratio is equal to a certain value $\hat{\sigma}$ (that we shall precise later), then the linear matter power spectrum $\mathrm{P}(\mathrm{k})$ differs from the "Cold Dark Matter" spectrum at all scales below a characteristic length (namely the damping scale) $l$, essentially determined by $\hat{\sigma}$. This means that there is a relationship between the damping scale $l$ and $\hat{\sigma}$, bearing in mind that $l$ has to be lower than the scale $l_{\text {struct }}$ of the smallest primordial structures that have been observed. What are the values of $\hat{\sigma}$ which induce cosmological effects in the linear matter power spectrum? Are those values possible in the case of light Dark Matter particles? This is what we shall now determine.

Since the elastic scattering cross sections of light Dark Matter candidates cannot be treated independently of the annihilation cross sections, we shall consider only values of the coupling constants, $m_{F}$ or $m_{U}$ that are compatible with relic density calculations. 


\subsection{Constraints on Dark Matter interactions}

In the picture commonly adopted nowadays, Dark Matter would belong to either the Cold or Warm Dark Matter scenarios. The latter is however less popular because of the lack of candidates and, presumably, because it relies on a free parameter, the Dark Matter mass. A lower limit on $m_{d m}$ can be obtained from the comparison of the free-streaming length with the scale $l_{\text {struct }}$. (Basically, $m_{d m} \geq 0.75 \mathrm{keV}$ preserves all scales beyond $l_{\text {struct }} \geq 0.16 \mathrm{Mpc}$ [36], but the WMAP's results now suggest that the values $m_{d m} \lesssim 0.75 \mathrm{keV}$ and even perhaps $\lesssim 10 \mathrm{keV}$ are ruled out [25,37], although this can be discussed too $[38])$.

In a realistic description, where one takes into account the Dark Matter interactions, the parameter $m_{d m}$ is associated with another parameter, namely the Dark Matter interaction rate. As a consequence, the free-streaming scale for the kind of particles we are interested in

- depends on both $m_{d m}$ and $\sigma_{e l}$ (the Dark Matter elastic scattering cross section that fixes its thermal decoupling);

- is supplemented by an additional damping length, namely the collisional damping scale, more directly related to Dark Matter interactions.

This additional scale can be split into two parts, namely a self-damping and induced-damping contributions, which respectively describe the influence of Dark Matter properties, and that of other species, on primordial fluctuations.

Because the induced damping actually corresponds to the damping acquired by a species $i$ (e.g. electrons, photons, or neutrinos) which is further transmitted to the Dark Matter fluctuations if the coupling $\mathrm{dm}-i$ is strong enough, there exists a relationship between the elastic cross section $\sigma_{e l(d m-i)}$, the Dark Matter mass and the scale $l$ at which the damping is expected to be of noticeable importance [34].

For the two species $i=\gamma, \nu$ which are expected to communicate the largest damping effects (and therefore the most stringent constraint on the cross section) $[34,35]$, this relationship can be displayed as:

$$
\sigma_{e l(d m-\nu)} \lesssim 310^{-35} \mathrm{~cm}^{2}\left(\frac{m_{d m}}{\mathrm{GeV}}\right)\left(\frac{l}{100 \mathrm{kpc}}\right)^{2}
$$

and

$$
\sigma_{e l(d m-\gamma)} \lesssim 610^{-34} \mathrm{~cm}^{2}\left(\frac{m_{d m}}{\mathrm{GeV}}\right)\left(\frac{l}{100 \mathrm{kpc}}\right)^{x}
$$


with $x \sim 1$, the damping scale being maximal when $l=l_{\text {struct }} \simeq 100 \mathrm{kpc}$. We then obtain $\hat{\sigma} \sim 310^{-35} \mathrm{~cm}^{2}\left(\frac{l}{100 \mathrm{kpc}}\right)^{2}$ when Dark Matter is coupled to neutrinos and $\hat{\sigma} \sim 610^{-34} \mathrm{~cm}^{2}\left(\frac{l}{100 \mathrm{kpc}}\right)$ when Dark Matter is coupled to photons.

The cross section (4) appears, at a first glance, very close to that obtained from the relic density condition, for particles with $m_{d m} \sim 10 \mathrm{MeV}$ and $l=100 \mathrm{kpc}$ (suggesting a surprising large damping effect) but one has to check that the elastic scattering cross section $\sigma_{e l(d m-\nu)}$ indeed behaves in the same way as the annihilation cross section $\sigma_{a n n}$.

The constraint on $\mathrm{dm}-\nu$ interactions comes from a new damping effect which can be seen as a mixing between the collisional damping and free-streaming effects [34]. It is such that any freely-propagating species $i(i=\nu$ in our case) can communicate its free-streaming damping to the Dark Matter fluctuations provided that the species $i$ happens to have interactions with Dark Matter which change the velocity of the Dark Matter fluid but not ${ }^{14}$ that of the fluid $i$ (say differently, such a coupling must not change the thermal decoupling of species $i$ ). The mixed damping is therefore at work only if the DM-neutrino interactions can decouple at a temperature below $\sim 1 \mathrm{MeV}$.

Equations (4) and (5) are given at specific times, namely the thermal decoupling of Dark Matter from neutrinos and photons (say $t_{d e c(d m-\nu)}$ and $t_{d e c(d m-\gamma)}$ respectively). If $\sigma_{e l(d m-\nu)}$ and $\sigma_{e l(d m-\gamma)}$, estimated in a given particle physics model, appear to be temperature dependent (i.e. $\left.\sigma_{e l(d m-i)} \propto T^{n}\right)$, then a more relevant constraint turns out to be given by the parameter $b_{e l}$, defined by $\sigma_{e l(d m-i)} c=b_{e l(d m-i)} T_{d e c(d m-i)}^{n}$, where $n$ is fixed by the model.

From now on, we shall focus on the neutrino induced-damping effect. We disregard the Dark Matter-photon elastic scattering cross section (based on box and triangle diagrams) in order to simplify our calculations. This one is expected to vanish with the photon energy $E_{\gamma}$, for $E_{\gamma}$ lower than the Dark Matter mass, due to gauge invariance and low energy theorems. We shall see that, within reasonable assumptions, the elastic scattering cross section of scalar Dark Matter particles with neutrinos at the Dark Matter thermal decoupling also appears to vanish with the neutrino energy (in fact it is proportional to the square of the neutrino energy, $\sigma_{e l(d m-\nu)} \propto T^{2}$, assuming Dark Matter is still in equilibrium with this species). The estimate of the neutrino induced damping effect is then expected to provide a damping scale either comparable or potentially larger than the photon constraint (although one should do in principle the comparison).

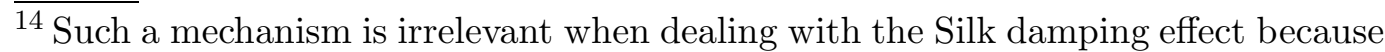
the baryons and photons thermally decouple (i.e. become collisionless) at the same time due to recombination.
} 
To estimate the neutrino induced-damping scale, we need first to express $b_{e l(d m-\nu)}$ explicitly. By using eq. (4) and the relationships between i) the cross section and the comoving interaction rate:

$$
\tilde{\Gamma}_{d e c(d m-i)} \simeq \sigma_{e l(d m-i)} c \tilde{n}_{d m} \frac{\rho_{i}}{\rho_{d m}} \simeq \sigma_{e l(d m-i)} c \tilde{n}_{i} \frac{T}{m_{d m}}, \quad \text { if } m_{d m}>T
$$

(with $\tilde{n}_{i}=n_{i} a^{3}$ ), ii) the comoving interaction rate and the scale factor in the radiation dominated era,

$$
\tilde{\Gamma}_{d e c(d m-i)} \approx 210^{-20} a_{\operatorname{dec}(d m-i)} \mathrm{s}^{-1},
$$

and finally iii) the scale factor and the temperature:

$$
a_{\operatorname{dec}(d m-i)} \simeq \frac{T_{0}}{T_{\operatorname{dec}(d m-i)}},
$$

one gets the relation:

$$
b_{e l} \approx 2.510^{28}\left(\frac{m_{d m}}{\mathrm{GeV}}\right)^{-1}\left(\frac{\sigma_{e l(d m-i)}}{\mathrm{cm}^{2}}\right)^{2} \mathrm{~cm}^{5} \mathrm{~s}^{-1},
$$

which turns into the following constraint:

$$
b_{e l(d m-\nu)} \lesssim 210^{-41}\left(\frac{l_{\text {struct }}}{100 \mathrm{kpc}}\right)^{4}\left(\frac{m_{d m}}{\mathrm{GeV}}\right) \mathrm{cm}^{5} \mathrm{~s}^{-1} .
$$

We now have to compute $b_{e l(d m-i)}$ in the case of light scalar Dark Matter candidates to determine whether they can induce changes in the linear matter power spectrum on visible scales.

\subsection{Elastic scattering cross section of scalar Dark Matter with neutrinos}

Once again, we shall assume that Dark Matter is not coupled to a fermion $F$ and a $U$ boson simultaneously. The elastic scattering cross section of non self-conjugate Dark Matter $\left(\mathrm{dm} \neq \mathrm{dm}^{\star}\right)$ with a (left handed) neutrino turns out to be given by:

$$
\sigma_{e l(\mathrm{dm}-\nu)} \approx \frac{C_{l}^{4}}{8 \pi m_{F}^{4}} T^{2}
$$

(within the local limit approximation for which $m_{d m} \ll m_{F}$ and assuming that the energy of the scattered neutrino is the same as that of the incoming one). In constrast, due to the relative sign between the $t$ and $u$-channel (see 
Appendix 9.3), the cross section $\sigma_{e l(\mathrm{dm}-\nu)}$ of self-conjugate scalar Dark Matter particles vanishes identically. Consequently, self-conjugate scalar candidates do not suffer from neutrino-induced damping effects at all.

A rough estimate indicates that $b_{e l} \approx 510^{-55} C_{l}^{4}\left(m_{F} / 100 \mathrm{GeV}\right)^{-4} \mathrm{~cm}^{5} \mathrm{~s}^{-1}$ for $\mathrm{dm} \neq \mathrm{dm}^{\star}$. If $m_{F} \sim 100 \mathrm{GeV}, C_{l} \approx 1$ and $1 \lesssim m_{d m} \lesssim 100 \mathrm{MeV}$, all the scales smaller than $\lesssim 10-0.3 M_{\odot}$ are damped respectively. Such a damping scale is actually too small to be constrained by present observations or tested by arguments on the reionization epoch $[32,38,37]$ so it might be very difficult to exclude such particles from their linear and non-linear matter power spectra. This collisional damping scale would be in fact even smaller if $C_{l} \ll 1$ or $m_{F} \gg 100 \mathrm{GeV}$. (One could consider, however, smaller values of $m_{F}$ provided one checks that they indeed evade limits notably on neutral supersymmetric particles.)

For comparison, the free-streaming scale (or, almost equivalently in this case, the self-damping scale), for particles which are likely to annihilate, behaves like $l_{f s} \approx 190 \mathrm{kpc}\left(\frac{m_{d m}}{1 \mathrm{MeV}}\right)^{-1 / 2}\left(\frac{a_{\operatorname{dec}(d m)}}{10^{-4}}\right)^{1 / 2}$ (if they thermally decouple in the radiation dominated era i.e. if $\left.a_{\operatorname{dec}(d m)}<10^{-4}\right)$. This expression is different from the well-known free-streaming formula because we are dealing with particles which have non-negligible interactions, i.e. for which the thermal decoupling epoch occurs after the non relativistic transition.

Before using this formula, one can check that light particles (with $m_{d m} \gtrsim$ $O(\mathrm{MeV})$ ) indeed thermally decouple after their non relativistic transition (so that they can potentially annihilate). The elastic cross section of non self-conjugate scalar particles with electrons (here seen to be the most relevant for determining the thermal decoupling epoch) is proportional to $\left(C_{l}^{2} C_{r}^{2} m_{e}^{2}\right) /\left(m_{d m}^{2} m_{F}^{2}\right)$. The decoupling is given by $a_{d e c}(d m-e)=510^{19}$ $\sigma_{e l(d m-e)} c \tilde{n}_{d m} \rho_{e} / \rho_{d m}$ which is equivalent to $a_{d e c(d m-e)} \approx 510^{19} \sigma_{e l(d m-e)} c \tilde{n}_{e}$ for $T_{d e c(d m-e)}>m_{d m}$ and to $a_{d e c(d m-e)} \approx 510^{19} \sigma_{e l(d m-e)} c \tilde{n}_{e} T_{d e c(d m-e)} / m_{d m}$ for $T_{d e c(d m-e)}<m_{d m}$ (here $\tilde{n}_{e}$ is the comoving electron number density which drops down once the electrons become non relativistic). With the above cross section, we find a decoupling temperature $T_{n r}<\mathrm{MeV}$. However, because electrons annihilate around $500 \mathrm{keV}$, the thermal decoupling of Dark Matter eventually occurs at a temperature close to $m_{e}$. Therefore, Dark Matter particles with $m_{d m}>O(\mathrm{MeV})$ should be able to annihilate so that their free-streaming scale should be indeed lower than $190 \mathrm{pc}$ (corresponding to $\approx 10 M_{\odot}$ ).

Thus, if light scalar Dark Matter particles are coupled to neutrinos through neutral fermions, both the free-streaming scale (or, similarly in fact, the selfdamping scale) and the neutrino-induced damping scale are negligible compared with the scale of primordial structures. 
These conclusions would have to be softened if there were a close degeneracy between $m_{d m}$ and $m_{F}$ (i.e. if $m_{F}^{2}-m_{d m}^{2}<2 m_{d m} E_{\nu}$ ) since, in this case, the elastic scattering cross section becomes roughly constant ${ }^{15}$ and one has to use the constraint given by eq.(4) instead of eq.(6). This appears more promising for large values of the Dark Matter mass $\left(m_{d m}>O(100) \mathrm{GeV}\right)$ because the case $m_{F} \sim m_{d m}$ cannot be excluded as yet, but this is, on the other hand, extremely unnatural because of the mass degeneracy it requires. Assuming, however, such a degeneracy (and $C_{l}=1, m_{d m} \sim 100 \mathrm{GeV}$ ), one finds a damping mass greater than $1 M_{\odot}$ but the required mass difference $m_{F}-m_{d m}$ is about a few hundred keV's! Without such a degeneracy, the linear $\mathrm{P}(\mathrm{k})$ associated with Dark Matter particles of $m_{d m} \sim 100 \mathrm{GeV}$ is expected to be damped much below $1 M_{\odot}$ (with $C_{l}=1$ and taking $m_{F} \sim 100$ $\mathrm{GeV}$ ), as the corresponding interactions decouple before $T \sim 1 \mathrm{MeV}$.

Let us now consider the elastic scattering cross section of scalar Dark Matter particles with neutrinos through the exchange of a $U$ boson. We find

$$
\sigma_{d m-\nu} c T_{d e c(d m-\nu)}^{-2} \approx 10^{-33} C_{U}^{2} f_{U_{l}}^{2}\left(\frac{m_{U}}{\mathrm{MeV}}\right)^{-4} \mathrm{~cm}^{5} \mathrm{~s}^{-1}
$$

Using $C_{U} f_{U_{l}} \simeq(3-12) 10^{-8}\left(m_{d m} / \mathrm{MeV}\right)^{-1}\left(m_{U} / \mathrm{MeV}\right)^{2}$, as imposed by relic density (and $g-2$ ) constraints, we merely see that $b_{e l(d m-\nu)}$ is about $b_{e l(d m-\nu)} \approx(1-15) 10^{-48}\left(m_{d m} / \mathrm{MeV}\right)^{-2} \mathrm{~cm}^{5} \mathrm{~s}^{-1}$. Thus, the neutrinoinduced damping in the case of a light gauge boson (and scalar Dark Matter) mainly depends on the Dark Matter mass. By comparing (6) with the above expression of $b_{e l(d m-\nu)}$, one finds that the damping scale associated with particles having a $m_{d m} \sim 4 \mathrm{MeV}$ for instance is about $310^{4} M_{\odot}$, if we assume that the coupling of the $U$ boson to neutrinos is the same as to electrons. (More precise values are derived in [39].) It may be reasonable, however, to consider that the $U$ boson does not couple to electrons and neutrinos with the same strength, especially in view of avoiding a too large contribution to the $\nu$ e elastic scattering cross section at low energy (which requires typically $\left|f_{U \nu} f_{U} / m_{U}^{2}\right| \lesssim G_{F}$ ), while keeping a sufficiently large value for the annihilation cross section of Dark Matter particles. In particular, decreasing the coupling of the $U$ boson to neutrinos would then tend (depending on the $U$ coupling to Dark Matter $C_{U}$ ) to make the damping mass even smaller than the above estimate. This damping scale gets, also, obviously smaller for larger Dark Matter masses.

To conclude this section, let us mention that the calculation of the elastic scattering cross section of light Dark Matter particles with neutrinos appears important when Dark Matter is coupled to a $U$ boson because the damping in

$\overline{15}$ The denominator, proportional to $m_{F}^{4}$, is indeed replaced by $m_{d m}{ }^{2} T^{2}$ which suppresses the dependence in $T^{2}$ in the numerator. 
the linear power spectrum is large enough to be quoted here. However, even for $m_{d m}<m_{\mu}$, it is still too small to modify the non linear matter power spectrum at cosmological scales. In fact, we expect that the smallest scales which are erased in the linear $\mathrm{P}(\mathrm{k})$ be regenerated once entered in the non linear regime; so the non linear matter power spectrum at $z=0$ and $10^{4} M_{\odot}$ might eventually appear similar to that in Cold Dark Matter scenarios [36,38], despite the unusual Dark Matter characteristics we are considering. In which case, it should be difficult to identify the nature of Dark Matter particles from the non linear and linear matter power and CMB spectra only.

\subsection{Damping scales of spin 1/2 candidates}

In the previous subsection, we found that the neutrino-induced damping scale of a scalar Dark Matter coupled to a fermion $F$ was null when Dark Matter is self-conjugate. We now estimate this collisional damping scale in the case of spin-1/2 Dark Matter candidates. Both the elastic scattering cross section of Majorana and Dirac spin-1/2 fermions are found to be proportional (in the

local limit) to $\sigma_{e l(\mathrm{dm}-\nu)} \propto \frac{C_{l}^{4}}{16 \pi m_{F}^{4}} T^{2}$. As expected, these cross sections are temperature (or neutrino energy) dependent $\left(\sigma_{e l(\mathrm{dm}-\nu)} c \simeq b_{e l(\mathrm{dm}-\nu)} T^{2}\right)$ so that the associated damping scale is given in terms of the parameter $b_{e l}$ (see eq. (6)). However, unlike scalars, the cross section for self-conjugate (i.e. here Majorana) particles does not vanish.

Because these cross sections are actually very close to that obtained with scalar Dark Matter, the collisional damping effect in the linear matter power spectrum should be of the same order of magnitude than what is found for scalar candidates (say $M \leq 10-0.3 M_{\odot}$ ) for $m_{F} \gtrsim 100 \mathrm{GeV}$ (and $m_{d m} \in[1-$ 100] MeV respectively). Also, for heavy Dark Matter particles (i.e. $m_{d m} \sim 100$ $\mathrm{GeV}$ ), one expects the damping scale to be negligible $M \ll 1 M_{\odot}$ unless there is an extremely close degeneracy, i.e. a few $\mathrm{keV}$, between $m_{F}$ and $m_{d m}$, in which case one gets a larger damping scale as a result of the enhancement of the neutrino-Dark Matter elastic scattering cross section. (If such a degeneracy exists, a more detailed study is required in order to take into account the coannihilations between $F$ and the Dark Matter particles.)

\section{$7 \quad$ Case of an asymmetry}

To satisfy the relic density requirement, we searched for the conditions which give rise to a Dark Matter annihilation cross section of the order of $\sigma_{\text {ann }} v_{\text {rel }} \sim$ $10^{-26}-10^{-27}\left(\Omega_{d m} h^{2} / 0.1\right) \mathrm{cm}^{3} \mathrm{~s}^{-1}$ in the unusual range $m_{d m}$ below a few 
GeV's. However, if there exists an asymmetry between the Dark Matter particle and antiparticle number densities (which may be necessary for $m_{d m} \lesssim$ $100 \mathrm{MeV}$, in the case of $F$ exchanges), the annihilation cross section can be in fact much larger than the value displayed previously (depending on the size of the asymmetry).

The question is then to determine whether larger values of the cross section are indeed possible within reasonable particle physics assumptions. We have seen that if Dark Matter was coupled to a new gauge boson, the mass $m_{U}$ and the couplings $C_{U}$ and $f_{U_{l}}, f_{U_{r}}$ should be small enough (for small values of $\left.m_{d m}\right)$ in order to get the proper value of the annihilation cross section. Larger values of $\sigma_{a n n} v_{r e l}$ can be obtained by increasing the coupling $C_{U}$ or decreasing $m_{U}$ but this requires an appropriate choice of parameters. On the other hand, scalar Dark Matter particles can naturally have a quite large annihilation cross section if they have non chiral couplings to a heavy fermion $F$ but its associated $g-2$ contribution should be cancelled by introducing a new set of particles. Once this "way out" accepted, one can indeed consider larger values of the couplings and justify an asymmetry between $n_{d m}$ and $n_{d m^{\star}}$, that will allow to evade the gamma ray constraint (which is crucial for $m_{d m} \lesssim 100$ $\mathrm{MeV})$.

For spin-1/2 Dirac fermions, on the other hand, it is rather difficult to consider such an asymmetry (unless $m_{d m}$ be larger than a few $\mathrm{GeV}$ ) because that would require values of the Yukawa couplings $C_{l}$ and $C_{r}$ which could potentially make the theory non perturbative.

In any case, increasing the couplings allows for larger Dark Matter elastic scattering cross sections with neutrinos and therefore larger neutrino-induced damping effects. However, if a spin-0 Dark Matter particle is coupled a fermion $F$, the maximum damping effect should be definitely lower than a few ten $M_{\odot}$ 's. If, on the other hand, Dark Matter is coupled to a $U$ boson, with $C_{U}$ larger than the values displayed in Appendix, then the corresponding damping scale could be in principle significantly larger than what is found without any asymmetry, but a more detailled study is required as other damping effects may then be at work. Imposing the condition $l \lesssim 100 \mathrm{kpc}$ could then perhaps constrain the maximum values of $C_{U}$ that is possible to consider.

\section{A possible candidate}

We have determined the conditions that allow for light scalar Dark Matter candidates to satisfy both the relic density and gamma ray constraints. They can be coupled to a heavy fermion (in which case an asymmetry between the particle and antiparticle number densities is required for $m_{d m} \lesssim 100 \mathrm{MeV}$ ), or to a new gauge boson (associated with, at least, an extra $U(1)$ symmetry). 


\subsection{A new spin-0 gauge boson as a possible Dark Matter candidate}

Now, we would like to point out that naturally stable spin-0 Dark Matter candidates coupled to fermions may be already be present within an existing class of theories or models which display, at least partly, an underlying $N=2$ extended supersymmetry [41]. Or also, in higher-dimensional theories, whether supersymmetry is present or not [42]. In both cases spin-1 gauge bosons are supplemented with new scalars which indeed appear as spin-0 "gauge" particles, so that we now expect, in these frameworks, the existence of new spin-0 gluon octets, or spin-0 photons, for example. The lightest of these spin-0 particles, if neutral and uncolored, may well be a viable Dark Matter candidate, along the lines discussed in the previous sections.

In such theories quarks and leptons are usually supplemented with new mirror partners. Indeed, in $N=2$ theories, quark and lepton fields belong to matter hypermultiplets (the latter describing 4-component Dirac fermion fields, i.e. left-handed as well as right-handed fields, both with the same gauge symmetry properties). Left-handed electroweak doublets and right-handed singlets associated with the usual quarks and leptons should then be accompanied by right-handed doublets and left-handed singlets. The latter would describe mirror partners $(F)$ of the ordinary quarks and leptons $(f)$. Such mirror partners also tend to be naturally present in higher-dimensional theories - whether or not they are supersymmetric - since 4d Dirac fermion fields with, initially, vectorial couplings to the gauge fields naturally appear as a result of the dimensional reduction.

Since none of these mirror particles has been observed yet, neither at LEP nor at TEVATRON, it is often believed that they simply don't exist at all, or at least disappear from the "low-energy" spectrum as the result of an appropriate supersymmetry-breaking (or dimensional-reduction) mechanism. We shall explore here the possibility according to which these mirror particles could indeed exist at not-too-high energies, say of the order of a few hundred $\mathrm{GeV} / \mathrm{c}^{2}$ 's, to fix the ideas. In a more specific way mirror neutrinos (with their righthanded component fields belonging to electroweak doublets) are coupled to $Z$ bosons and should certainly be heavier than about $40 \mathrm{GeV} / c^{2}$, otherwise they should have been produced in $Z$ decays at LEP. Mass splittings within $S U(2)$ doublets of mirror particles also should not be too large, otherwise they could generate a too large contribution to the $\rho$ parameter of the electroweak theory.

Just as one expects, in ordinary supersymmetric theories, the existence of a "lightest supersymmetric particle" which may naturally be stable thanks to $R$ parity, one may also expect here the stability of some "lightest mirror particle", in relation with a $Z_{2}$ discrete symmetry $M_{p}$ under which mirror fermions (and 
sfermions, within supersymmetric theories) would be odd. This symmetry may be referred to as "mirror-parity", provided one carefully remembers that it does not exchange ordinary with mirror particles, but simply changes the signs of the fields describing the latter. New spin-0 gauge particles, which in these extended SUSY or higher-dimensional theories couple ordinary to mirror fermions, would then also be odd under this discrete $M_{p}$ symmetry operator. The "lightest $M$-odd particle" (or LMP) is then expected to be absolutely stable, if indeed $M$-parity is conserved, and a potential Dark Matter candidate.

This LMP could be a mirror neutrino (in which case it should be heavy) or one of the new neutral spin-0 gauge bosons, which may well be light, the case of interest to us here. We would then expect mirror particles to have decay modes such as:

$$
\left\{\begin{array}{l}
l_{\text {Mirror }} \rightarrow l+\text { new spin-0 gauge particle } \\
q_{\text {Mirror }} \rightarrow q+\text { new spin-0 gauge particle }
\end{array}\right.
$$

The lightest of the new "gauge scalars" could then be absolutely stable, if lighter than all mirror fermions. It could be a spin- 0 photon ${ }^{16}$ or, maybe more plausibly, one of the spin-0 partners of the weak hypercharge $U(1)$ gauge field $B^{\mu}$. (Note that these neutral gauge scalars, which have electroweak quantum numbers $T_{3}=Y=0$, are not coupled to the $Z$ boson so that they cannot be directly produced in $Z$ decays at LEP.)

The pair production of unstable mirror particles would then ultimately lead to missing energy-momentum (precisely carried away by two unobserved spin-0 photons or more generally neutral spin-0 gauge particles), just as in supersymmetry, a signature of the pair production of SUSY particles is missing energy-momentum carried away by two unobserved photinos or neutralinos, the "lightest SUSY particle" being stable.

It is also possible (at least in principle) to consider a continuous $M$-symmetry instead of a $M$-parity (related to $M_{p}$ by $M_{p}=(-1)^{M}$ ). The corresponding lightest stable particle would then carry plus (or minus) one unit of an additive conserved quantum number $M$, and would be described by a complex field $\frac{a-i b}{\sqrt{2}}$. This particle would then differ from its antiparticle, both being stable but carrying opposite values $( \pm 1)$ of the conserved additive quantum number $M$. This situation may be particularly interesting in the presence of an initial

\footnotetext{
${ }^{16}$ Although a mirror neutrino is not directly coupled to a spin-0 photon, it could still decay into $\nu+$ spin-0 photon through radiative effects, or have other decay modes like $\nu_{\text {Mirror }} \rightarrow \nu e^{+} e^{-}+$spin-0 photon. The lightest spin- 0 mass eigenstate may also turn out to be a spin-0 partner of the weak hypercharge gauge field $B^{\mu}$, in which case it directly couples mirror neutrinos to ordinary ones.
} 
asymmetry between the corresponding numbers of particles and antiparticles, whether this asymmetry has been present from the beginning, or induced by $M$-violating interactions at some point in the evolution of the Universe. The relic abundance of the lightest, stable, $M=+1$ particle may then be determined essentially by the size of the particle/antiparticle asymmetry (provided one can achieve a sufficiently large value of the annihilation cross-section), as it is believed to happen for the relic abundance of baryons in the Universe. This is especially relevant for light Dark Matter candidates, which could both acquire the correct relic density while at the same time - in the presence of such an aymmetry - avoiding the gamma ray constraint which applies to candidates lighter than about $100 \mathrm{MeV}$, as discussed in Section 7. This would require, however, the above $M$-symmetry to survive unbroken down to low energies, a requirement which tends to be in conflict with the necessity of generating masses for both ordinary fermions $f$ and mirror fermions ${ }^{17} F$.

Finally, we also note that the chiral (or non chiral) character of the couplings of such new spin-0 Dark Matter particles to fermion/mirror-fermion pairs is generally related with the complex (or real) character of the corresponding fields; but that mixing effects associated with mechanisms of mass generation and symmetry breaking may also generate non chiral couplings from couplings which were, initially, of a chiral nature. This is essential in the phenomological analysis, as we saw earlier. We should of course be attentive to the fact that the requirement of non chiral couplings (in view of sufficiently large annihilation cross sections allowing for a suitable value of the relic abundance) has to be reconciled with the constraints from the anomalous magnetic moments of charged leptons (as discussed in Appendix 9.4).

\subsection{Extended supersymmetry}

Furthermore, in an extended SUSY framework (possibly although not necessarily associated with a higher-dimensional supersymmetric theory) we are led, through the consideration of two $Z_{2}$ discrete symmetries, to a natural framework for two neutral stable particles, e.g. a rather familiar neutralino LSP plus a spin-0 photon (or companion of the weak hypercharge gauge field $\left.B^{\mu}\right)$ LMP. Depending on the values of their masses and interaction rates, they may turn out to be acceptable dark matter candidates, under the conditions discussed earlier.

Let us recall first some important features of $N=2$ extended supersymmetric theories [41]. When these are formulated with an $N=1$ superfield formalism,

\footnotetext{
17 This concerns only a continous $M$-symmetry, not the discrete symmetry of $M$ parity, which in any case governs the stability of the Dark Matter candidate considered here, whether or not they are their own antiparticles.
} 
ordinary gauge superfields are accompanied by additional $(N=1)$ "chiral gauge superfields", so as to describe, jointly, $N=2$ massless gauge multiplets. At the same time matter (and also Higgs) chiral superfields systematically occur in pairs, so as to describe $N=2$ "hypermultiplets". $N=2$ gauge multiplets describe in particular two gluino octets, two photinos, ... as well as two color-octets of spin-0 gluons, two spin-0 photons, etc.:

\begin{tabular}{|l|c|c|}
\hline photon & 2 spin- $\frac{1}{2}$ photinos & 2 spin-0 photons \\
gluons & 2 spin- $\frac{1}{2}$ gluino octets & 2 spin-0 gluon octets \\
\hline
\end{tabular}

These particles all remain massless, as long as the $N=2$ supersymmetry is kept unbroken. These new "scalar gauge fields" may also appear as originating from the fifth and sixth components of higher-dimensional gauge fields $V^{\hat{\mu}}$, in a six-dimensional spacetime [42]. Each pair of gaugino fields in 4 dimensions then originates from a single 8-component chiral gaugino field, in a 6-dimensional spacetime.

As we have seen extended supersymmetric theories, however, have the unpleasant feature of naturally leading to vectorlike and even vectorial theories, necessitating the introduction of chirality-breaking mechanisms for matter fields, and of mechanisms responsible for quark and lepton masses. This led us to consider mirror lepton and quark fields. Even if we are led, at a certain point, to abandon the full $N=2$ supersymmetry in favor of a simple $N=1$ subalgebra (or even of no supersymmetry at all), it is still conceivable that some sectors (e.g. gauge and Higgs bosons, together with their superpartners) could display visible signs of the underlying extended supersymmetry. Whether this is indeed the case or not, however, does not affect significantly our present analysis of possible Dark Matter candidates.

The $S U(3) \times S U(2) \times U(1)$ gauge group may be spontaneously broken into the $S U(3) \times U(1)$ subgroup of QCD $\times$ QED, with the help of - at least two electroweak Higgs doublet fields, which are described in the $N=1$ superfield formalism by the familiar $H_{1}$ and $H_{2}$ chiral superfields, as usual in the (M)SSM. It is however more elegant to use a quartet of spin-0 Higgs doublets. After the electroweak symmetry breaking the $W^{ \pm}$and $Z$ massive gauge bosons then belong to complete charged and neutral massive gauge multiplets of the extended $N=2$ supersymmetry. These multiplets also describe 4 Dirac winos (or 4 Majorana zinos) appearing as gaugino-higgsino mixtures, as well as 5 charged (or 5 neutral) spin-0 Higgs bosons (i.e. altogether 32 degrees of freedom for the massive gauge multiplet of the $W^{ \pm}$, and 16 for the $Z$ boson) [41]. All these particles have the same mass $m_{W}$, or $m_{Z}=m_{W} / \cos \theta$, as long 
as the $N=2$ supersymmetry is kept unbroken. In the harmonic superspace formalism appropriate to the description of $N=2$ extended supersymmetry, all four doublet Higgs fields may be described by a single $N=2$ electroweak doublet Higgs superfield

$$
\omega=\left(\begin{array}{c}
\omega^{0} \\
\omega^{-}
\end{array}\right) .
$$

Its charged component as well as the imaginary part of its neutral component get eliminated, or "eaten away", while the $N=2$ gauge superfields describing the $W^{ \pm}$and $Z$ acquire masses [43]. The remaining uneaten real part of $\omega^{0}$ describes 4 neutral spin-0 Higgs bosons accompanied by two Majorana higgsinos.

Whether the extra spin-0 components of gauge fields should actually show up or not in the low-energy theory depends on the details of the mechanism that should be responsible for the breaking of the extended supersymmetry (and/or the compactification of the extra space dimensions). The breaking of the supersymmetry may be elegantly obtained by demanding periodic and antiperiodic boundary conditions for ordinary $R$-even particles and their $R$ odd superpartners, respectively - in which case the masses of the (lowestlying) gravitinos, gluinos and photinos, which fix the energy scale at which supersymmetric particles should start to show up, would be given, in the simplest case and up to radiative correction effects, by

$$
m_{3 / 2}=m_{1 / 2}=\frac{\pi \hbar}{L c}=\frac{\hbar}{2 R c}
$$

in terms of the size $L$ of the extra dimension responsible for supersymmetry breaking (or of the corresponding "radius" $R$ ). This led us to consider the possibility of relatively "large" extra dimensions, associated with a compactification scale that could then be as "low" as $\sim$ TeV scale[44]. In such a framework, widely discussed now, it is quite conceivable that the new spin-0 states, as well as mirror lepton and quark fields, may only manifest themselves at the compactification scale. On the other hand, it is also legitimate to discuss their physical effects, should they actually be present in the low-energy theory, much below the compactification scale.

\subsection{Two discrete symmetries}

The new $M$-odd spin- 0 states are expected to couple ordinary matter (lepton and quark) fields to mirror lepton and quark fields, as discussed earlier. We 
do not discuss here the mechanism by which ordinary as well as mirror lepton and quark fields should acquire their masses, which may lead us to abandon, at some point, the full $N=2$ supersymmetry. Just as a $N=1$ supersymmetric theory may admit a discrete $R$-parity symmetry - which is a discrete $Z_{2}$ remnant of a continuous $R$-symmetry, with $R_{p}=(-1)^{R}$ - an extended $N=2$ theory in general admits an extended $S U(2)$ or $S U(2) \times U(1)$ global $R$ symmetry group, acting on the doublet of supersymmetry generators,

$$
\left(\begin{array}{l}
Q_{L}^{1} \\
Q_{L}^{2}
\end{array}\right)
$$

One can then initially define two distinct $R$-parity symmetry operators, $R_{1 p}$ and $R_{2 p}$, associated with the first and second supersymmetry generators, respectively, the usual $R$-parity symmetry then corresponding to the product

$$
R_{p}=R_{1 p} R_{2 p}
$$

The $R_{1}$ and $R_{2}$ parities within the $N=2$ massless gauge multiplets and matter multiplets are given as $\left|R_{1 p} R_{2 p}\right\rangle$ in the Table below, together with the corresponding values of $R$-parity. By singling out one of the two supersymmetry generators (say $Q_{1}$ ), we may also redefine one of the two $R_{i p}$ discrete symmetries (say $R_{2 p}$ ) as the previously discussed mirror-parity symmetry $M_{p}$, a point to which we shall return soon.

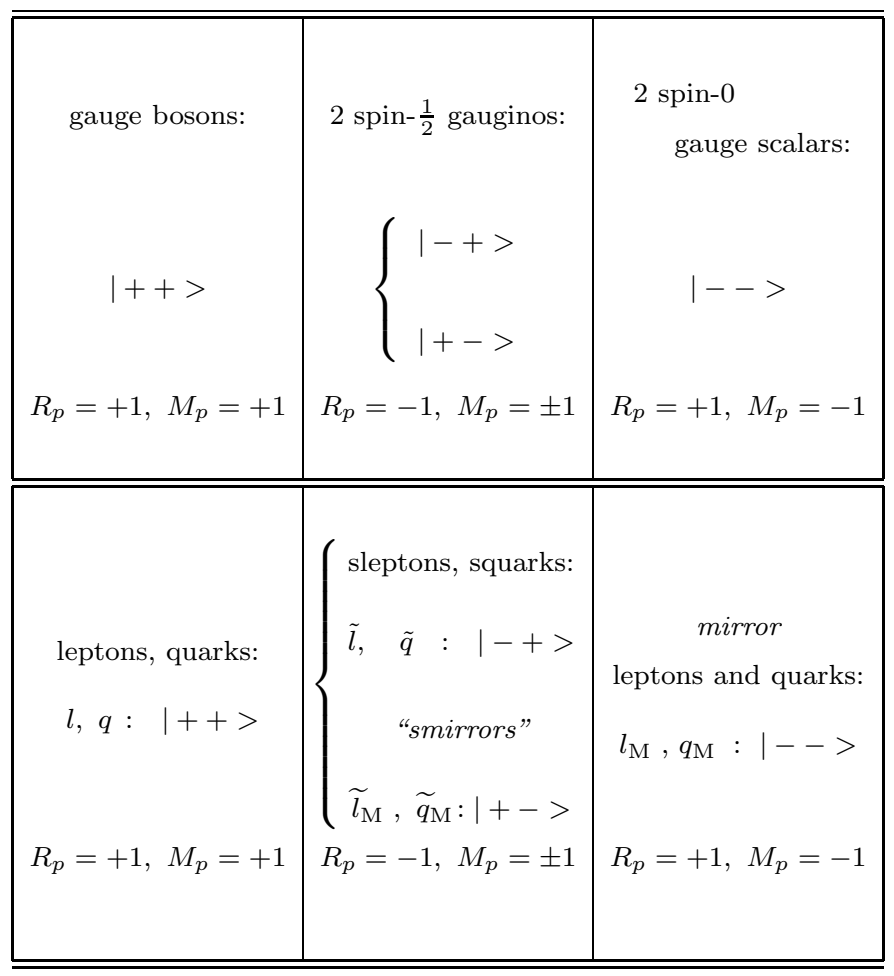


Among the four electroweak Higgs doublets which tend to be naturally present in such theories, which all have $R$-parity equal to +1 (with $\mid R_{1 p} R_{2 p}>=$ $|++>|+,+>, \mid-->$ and $\mid-->)$, two, which acquire non-vahishing v.e.v.'s, may be defined so as to have $M$-parity (identified as $R_{2 p}$ ) equal to +1 , while the two others (which keep vanishing v.e.v.'s) have $M$-parity equal to -1 ; the spontaneous breaking of the electroweak symmetry keeping intact the discrete mirror-parity symmetry, as well as the usual $R$-parity.

\subsection{Two stable Dark Matter candidates, from extended supersymmetry}

Once we have chosen to restrict the full $N=2$ supersymmetry of the initial theory to a simple and familiar $N=1$ supersymmetry, the $R_{2 p}$ discrete $Z_{2}$ symmetry may also be identified to the "mirror-parity" operator that we denoted previously as $M_{p}$. It commutes with the first SUSY generator $\left(Q_{1}\right)$ and is equal to +1 for the ordinary particles of the supersymmetric standard model, including those described by the two doublet Higgs superfields $H_{1}$ and $H_{2}$. $M$-parity, on the other hand, is equal to -1 for the mirror particles, their superpartners, and for the spin-0 gauge particles which couple ordinary leptons and quarks to their mirror partners, together with the new inos (second octet of gluinos, additional charginos, neutralinos, and Higgs bosons) necessitated by the original extended supersymmetry.

The first two Higgs doublets, with $M_{p}=+1$, correspond to the spin- 0 components of the familiar $H_{1}$ and $H_{2}$ superfields of the (M)SSM, responsible for lepton and quark masses. The same Higgs doublets described by $H_{1}$ and $H_{2}$ could also be responsible for very heavy mirror lepton and quark masses (should such particles effectively be present in the low-energy theory), if one assumes large Yukawa couplings to mirror particles, without generating a breaking of the discrete $M$-parity symmetry. In this framework mirror quark and lepton masses - which occur in violation of the $S U(2) \times U(1)$ electroweak gauge symmetry are expected to be smaller than a few hundred $\mathrm{GeV} / c^{2}$ 's (unless one is ready to tolerate very large values of Yukawa coupling constants, leading to mirror particles significantly heavier than the $W^{ \pm}$and $Z$ 's). Given the limited success, within ordinary supersymmetry, to find attractive and predictive models of supersymmetry-breaking, we should leave open the range of possibilities for the masses of the new particles. This allows us, in particular, to pay special phenomenological attention to the possibility of a new light stable spin-0 Dark Matter particle, as we have done here.

\section{Conclusion}

In this paper, we discussed the possibility that scalar particles as light as a few

$\mathrm{MeV}$ (and no diagonal coupling to the $Z$ boson) could be a viable explanation 
to the Dark Matter issue. In particular, we determined the conditions for the mass range $[O(\mathrm{MeV})-O(\mathrm{GeV})]$ to simultaneously satisfy particle physics, relic density and gamma ray constraints. We found that:

- Scalar Dark Matter particles coupled to heavy fermions $F\left(m_{F}>100 \mathrm{GeV}\right)$ can have an annihilation cross section into ordinary fermions large enough to give rise to the observed value of $\Omega_{d m} h^{2}$ but this requires the coupling $\mathrm{dm}-\bar{f}$ $F$ to be non chiral. However, when this condition is satisfied, the production of gamma rays associated with light particles $\left(m_{d m} \lesssim 100 \mathrm{MeV}\right)$, in the energy range below one hundred MeV's, is found to be much larger than what is observed. Thus, in order to maintain light particles as a viable solution to the Dark Matter problem, there should exist an asymmetry between their particle and antiparticle number densities. On top of that, one may also have to introduce additional particles (e.g. charged scalars and neutral fermions or a pseudoscalar particle), to cancel the too large contribution $a_{\mu, e}^{m_{F}}$ that is required to get the desired value of the annihilation cross section. The new particles we introduce are expected to be within the reach of future LHC experiments, so it should be possible to test and maybe to exclude the possibility of light scalar Dark Matter particles in a close future.

The range $m_{d m}>O(100 \mathrm{MeV})$, on the other hand, does not require any asymmetry assumption and appears much easier to test and presumably to rule out, notably through gamma ray predictions (if this asymmetry does not exist indeed) or from various experiments, like direct Dark Matter searches (for $m_{d m}>\mathrm{GeV}$ ).

- Scalar Dark Matter particles coupled to a new gauge boson $U$ have an annihilation cross section naturally proportional to $v_{d m}^{2}$ at the freeze-out epoch. Therefore, the gamma ray constraint appears much easier to achieve for candidates with $m_{d m} \lesssim 100 \mathrm{MeV}$. Since, on the other hand, their annihilation cross section turns out to depend significantly on the Dark Matter mass (as in the case of fermionic Dark Matter), one can get an acceptable relic density provided the $U$ boson is light enough and the couplings sufficiently small (when Dark Matter is light). Basically, to satisfy both the relic density requirement and the gamma ray constraints, one can take, for instance, $m_{d m} \sim 4 \mathrm{MeV}, m_{U} \sim 10 \mathrm{MeV}, C_{U} \sim 410^{-3}$ and $f_{U_{l}} \sim 410^{-4}$. These values, directly compatible with the electron and muon $g-2$ constraints, correspond to a $U$ boson lifetime of the order of $\approx 10^{-15} \mathrm{~s}$.

If coupled to a $U$ boson, these light Dark Matter particles $\left(m_{d m} \lesssim 100\right.$ $\mathrm{MeV}$ ) should yield a gamma ray flux lower than $10^{-5}\left(\frac{m_{d m}}{\mathrm{MeV}}\right)^{-2} \mathrm{~cm}^{-2} \mathrm{~s}^{-1}$, which seems close (for $m_{d m}=1 \mathrm{MeV}$ ) but still out of the reach of present gamma ray experiments for $m_{d m}>O(\mathrm{MeV})$. However, a gammay ray signature from the galactic centre at low energy could be due to the anni- 
hilation of such light Dark Matter particles. And residual annihilations in structures at "large redshift" could perhaps yield reionizing photons that might influence structure formation. Thus light Dark Matter particles coupled to a $U$ boson might be possible and quite interesting regarding their implications on structure formation.

In conclusion, we found two different scenarios in which Dark Matter could be made of light scalar particles. The first one relies on the existence of heavy (charged) fermionic particles $F$ (e.g. mirror fermions) and presumably other particles to cancel $g-2$ contributions, and the second one, on the existence of a light gauge boson. There might exist other scenarios that allow for light scalar candidates (e.g. with a dominant coupling to quarks or couplings with a light neutrino of a new kind) but we did not explore them. To end, we mentioned a possible framework, originating from extended supersymmetry and/or extra spacetime dimensions, in which scalar particles would appear as natural Dark Matter candidates.

\section{Acknowledgments}

The authors would like to thank T. Abel, J. Collins, J. Devriendt, K. Hamilton, J. Iliopoulos, J. Silk, D. Spergel, M. Strikman and J. Taylor for helpful discussions. C.B. is supported by an individual PPARC Fellowship.

\section{Appendix}

We now give the expressions of the squared matrix elements associated with the cross sections that enter the estimate of the relic density and damping scales of Dark Matter candidates. We disregard the annihilations into two photons, expected to be negligible compared with annihilations into a pair fermion-antifermion. We also disregard the annihilations into $W^{+} W^{-}$or $Z Z$ only relevant for $m_{d m} \geq m_{W}, m_{Z}$ respectively.

We shall consider only tree-level processes where either a $F$ or $U$ boson is exchanged, disregarding possible interference terms which are not expected to modify our conclusions. 


\subsection{Annihilation cross sections associated with the exchange of a $F$}

All expressions regarding Dark Matter annihilations are given in terms of the inverse of the squares of the $t$-channel and $u$-channel denominators, developed as:

$$
\begin{aligned}
& \left(t-m_{F}^{2}\right)^{-2}=T_{0}+T_{1} p_{d m} \cos \theta+p_{d m}^{2}\left(T_{20}+T_{21} \cos \theta^{2}\right) \\
& \left(u-m_{F}^{2}\right)^{-2}=T_{0}-T_{1} p_{d m} \cos \theta+p_{d m}^{2}\left(T_{20}+T_{21} \cos \theta^{2}\right)
\end{aligned}
$$

and

$$
\left(\left(t-m_{F}^{2}\right)\left(u-m_{F}^{2}\right)\right)^{-1}=T_{0}+p_{d m}^{2}\left(T_{20}+T_{21} \cos \theta^{2} / 3\right)
$$

with

$$
\begin{aligned}
& T_{0}=\frac{1}{\left(-m_{F}^{2}-m_{d m}^{2}+m_{f}^{2}\right)^{2}}, \\
& T_{1}=\frac{-4 \sqrt{m_{d m}^{2}-m_{f}^{2}}}{\left(-m_{F}^{2}-m_{d m}^{2}+m_{f}^{2}\right)^{3}}, \\
& T_{20}=\frac{4}{\left(-m_{d m}^{2}+m_{f}^{2}-m_{F}^{2}\right)^{3}}, \\
& T_{21}=\frac{12\left(m_{d m}^{2}-m_{f}^{2}\right)}{\left(m_{d m}^{2}-m_{f}^{2}+m_{F}^{2}\right)^{4}} .
\end{aligned}
$$

In the following, we compute and display the quantity

$$
|\mathcal{M}|^{2}=\int_{-1}^{+1}|M|^{2} d \cos \theta_{c m}
$$

where $|M|^{2}$ is estimated in the centre of mass reference frame. With these conventions, the annihilation cross section times velocity is related to $|\mathcal{M}|^{2}$ by:

$$
\sigma_{\text {ann }} v_{r e l}=\frac{1}{16 \pi s} \sum_{s_{i}, s_{j}} \frac{1}{\left(2 s_{i}+1\right)\left(2 s_{j}+1\right)}|\mathcal{M}|^{2}
$$

$\sum_{s_{i}, s_{j}}$ denoting the usual sum over the spins of the incoming particles. Let us start with the annihilation of spin-1/2 Majorana fermions. 


\subsubsection{Majorana Dark Matter}
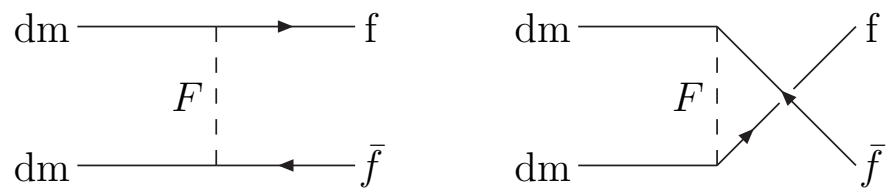

$$
\begin{aligned}
|\mathcal{M}|^{2}= & 8 T_{0} m_{d m}^{2}\left(2 C_{l} C_{r} m_{d m}+\left(C_{l}^{2}+C_{r}^{2}\right) m_{f}\right)^{2} \\
& +\frac{8 p_{d m}^{2}}{3}\left[2 T _ { 0 } \left(2 m_{d m}^{2}\left(2 C_{l}^{4}+9 C_{l}^{2} C_{r}^{2}+2 C_{r}^{4}\right)\right.\right. \\
& \left.\quad+6 C_{l} C_{r}\left(C_{l}^{2}+C_{r}^{2}\right) m_{d m} m_{f}-\left(C_{l}^{4}+12 C_{l}^{2} C_{r}^{2}+C_{r}^{4}\right) m_{f}^{2}\right) \\
& -4 T_{1}\left(C_{l}^{2}+C_{r}^{2}\right) m_{d m} \sqrt{m_{d m}^{2}-m_{f}^{2}}\left(\left(C_{l}^{2}+C_{r}^{2}\right) m_{d m}+2 C_{l} C_{r} m_{f}\right) \\
& +3 m_{d m}^{2} T_{20}\left(2 C_{l} C_{r} m_{d m}+\left(C_{l}^{2}+C_{r}^{2}\right) m f\right)^{2} \\
& +m_{d m}^{2} T_{21}\left(4\left(C_{l}^{4}+3 C_{l}^{2} C_{r}^{2}+C_{r}^{4}\right) m_{d m}^{2}\right. \\
& \left.\left.+20 C_{l} C_{r}\left(C_{l}^{2}+C_{r}^{2}\right) m_{d m} m_{f}+\left(C_{l}^{4}+18 C_{l}^{2} C_{r}^{2}+C_{r}^{4}\right) m_{f}^{2}\right) / 3\right] .
\end{aligned}
$$

Our expression is consistent with that displayed in [40] for Bino-like particles. In particular, one readily sees that the S-wave annihilation cross section of two Majorana spin-1/2 particles into an ordinary fermion-antifermion pair is suppressed proportionately to $m_{f}^{2} / m_{F}^{4}$ (in the local limit approximation) in the case of chiral couplings $\left(C_{l} C_{r}=0\right)$ while both the $v_{d m}$ dependent and independent terms turn out to be comparable if one allows for non chiral couplings. This is not expected to bring any drastic changes in the case of a light Dark Matter particle $\left(m_{d m}<O(\mathrm{GeV})\right)$ because of the small mass difference between $m_{d m}$ and $m_{f}$ but this may be more interesting in supersymmetry, when the lightest neutralino is heavier than the top quark $\left(m_{\chi}>m_{t}\right)$ and there exists a mixing angle between the two stop eigenstates $\left(\tilde{t}_{l}\right.$ and $\left.\tilde{t}_{r}\right)$. 


\subsubsection{Dirac Dark Matter}

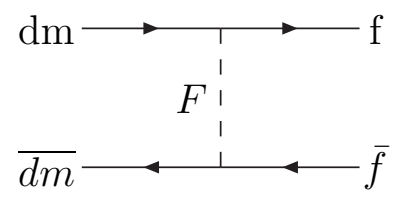

In the case of spin-1/2 Dirac fermions, we find:

$$
\begin{aligned}
& |\mathcal{M}|^{2}=8 T_{0} m_{d m}^{2}\left(\left(C_{l}^{2}+C_{r}^{2}\right) m_{d m}+2 C_{l} C_{r} m_{f}\right)^{2} \\
& +\frac{8 p_{d m}^{2}}{3}\left[T_{0}\left(C_{l}^{2}+C_{r}^{2}\right)\right. \\
& \left(7\left(C_{l}^{2}+C_{r}^{2}\right) m_{d m}^{2}+12 C_{l} C_{r} m_{d m} m_{f}-\left(C_{l}^{2}+C_{r}^{2}\right) m_{f}^{2}\right) \\
& -2 T_{1} m_{d m}\left(C_{l}^{2}+C_{r}^{2}\right) \sqrt{m_{d m}^{2}-m_{f}^{2}}\left(\left(C_{l}^{2}+C_{r}^{2}\right) m_{d m}+2 C_{l} C_{r} m_{f}\right) \\
& \left.+\left(3 T_{20}+T_{21}\right) m_{d m}^{2}\left(\left(C_{l}^{2}+C_{r}^{2}\right) m_{d m}+2 C_{l} C_{r} m_{f}\right)^{2}\right] .
\end{aligned}
$$

Both the $\mathrm{S}$ and $\mathrm{P}$-wave contributions (or more precisely the velocity dependent and independent terms) are now dominated by $m_{d m}^{2} / m_{F}^{4}$ (in the local limit) when $C_{l} C_{r}=0$. This is in contrast with spin-1/2 Majorana fermions where the $\mathrm{S}$-wave is found to be proportional to $m_{f}^{2} / m_{F}^{4}$ (in the same situation), indicating that Dirac and Majorana spin-1/2 particles do not behave in the same way.

\subsubsection{Self-conjugate scalar Dark Matter}
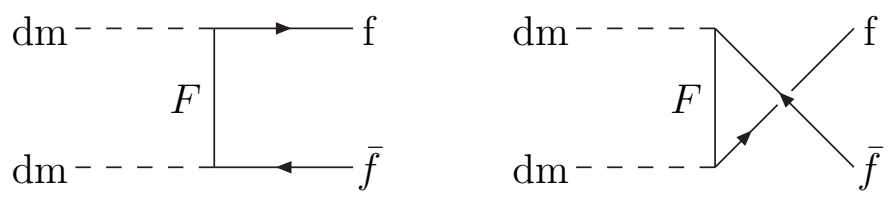

In the case of the annihilation of two self-conjugate scalars, one finds:

$$
|\mathcal{M}|^{2}=16 T_{0}\left(\left(C_{l}^{2}+C_{r}^{2}\right) m_{f}+2 C_{l} C_{r} m_{F}\right)^{2}\left(m_{d m}^{2}-m_{f}^{2}\right)
$$




$$
\begin{gathered}
\quad+\frac{16}{9} p_{d m}^{2}\left(\left(C_{l}^{2}+C_{r}^{2}\right) m_{f}+2 C_{l} C_{r} m_{F}\right) \\
{\left[9\left(\left(C_{l}^{2}+C_{r}^{2}\right) m_{f}+2 C_{l} C_{r} m_{F}\right) T_{0}-3 T_{1}\left(C_{l}^{2}+C_{r}^{2}\right) m_{f} \sqrt{m_{d m}^{2}-m_{f}^{2}}\right.} \\
\left.\quad+\left(9 T_{20}+2 T_{21}\right)\left(\left(C_{l}^{2}+C_{r}^{2}\right) m_{f}+2 C_{l} C_{r} m_{F}\right)\left(m_{d m}^{2}-m_{f}^{2}\right)\right] .
\end{gathered}
$$

The S-wave contribution is seen to be proportional to $1 / m_{F}^{2}$ when the couplings are non chiral (instead of $m_{f}^{2} / m_{F}^{4}$ as obtained for Majorana and $m_{d m}^{2} / m_{F}^{4}$ for Dirac particles). The corresponding cross section then appears almost independent of the Dark Matter mass, suggesting that it should be possible to get the correct relic density for light Dark Matter particles.

\subsubsection{Non self-conjugate scalar Dark Matter}

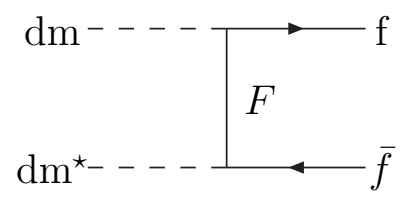

The squared amplitude associated with the annihilation of non self-conjugate scalars into a fermion and antifermion pair is given by:

$$
\begin{aligned}
& |\mathcal{M}|^{2}=4 T_{0}\left(m_{d m}^{2}-m_{f}^{2}\right)\left(\left(C_{l}^{2}+C_{r}^{2}\right) m_{f}+2 C_{l} C_{r} m_{F}\right)^{2} \\
& +\frac{4 p_{d m}^{2}}{3}\left[-\left(\left(C_{l}^{2}+C_{r}^{2}\right) m_{f}+2 C_{l} C_{r} m_{F}\right)\right. \\
& \begin{array}{r}
\left(2 T_{1}\left(C_{l}^{2}+C_{r}^{2}\right) m_{f} \sqrt{m_{d m}^{2}-m_{f}^{2}}+\right. \\
\left.\left(3 T_{20}+T_{21}\right)\left(-m_{d m}^{2}+m_{f}^{2}\right)\left(\left(C_{l}^{2}+C_{r}^{2}\right) m_{f}+2 C_{l} C_{r} m_{F}\right)\right) \\
+2 T_{0}\left(6 C_{l} C_{r}\left(C_{l}^{2}+C_{r}^{2}\right) m_{f} m_{F}+\left(C_{l}^{4}+C_{r}^{4}\right)\left(2 m_{d m}^{2}+m_{f}^{2}\right)\right. \\
\left.\left.+6 C_{l}^{2} C_{r}^{2}\left(m_{f}^{2}+m_{F}^{2}\right)\right)\right] .
\end{array}
\end{aligned}
$$

Here, both the $\mathrm{S}$ and $\mathrm{P}$-wave contributions are proportional to $1 / m_{F}^{2}$ in the case of non chiral couplings. Whether the couplings are chiral or not, our expressions for self-conjugate and non self-conjugate scalars are similar so the 
assumption $d m=d m^{*}$ or $d m \neq d m^{*}$ is not crucial concerning the derivation of the annihilation cross section of scalar particles.

9.2 Annihilation cross sections associated with the production of a $U$ boson

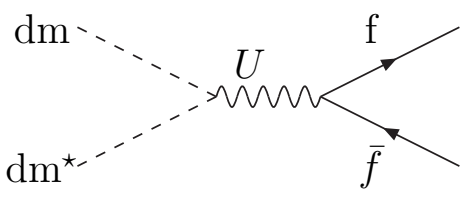

The cross section corresponding to the virtual $s$-channel production of a $U$ boson is seen to be given by:

$$
|\mathcal{M}|^{2}=\frac{16 C_{U}^{2} p_{d m}^{2}}{3\left(m_{U}^{2}-4 m_{d m}^{2}\right)^{2}}\left[4 m_{d m}^{2}\left(f_{U_{l}}^{2}+f_{U_{r}}^{2}\right)-m_{f}^{2}\left(f_{U_{l}}^{2}-6 f_{U_{l}} f_{U_{r}}+f_{U_{r}}^{2}\right)\right]
$$

This cross section is very small when the $U$ boson is very heavy but it is, on the other hand, of the good order of magnitude when the $U$ boson is light enough, thereby allowing for light Dark Matter to satisfy the relic density condition (in which case, the $U$ boson should be very weakly coupled).

\subsection{Elastic scattering cross sections of Dark Matter with neutrinos}

Let us now derive the square of the matrix element associated with the elastic scattering cross section of Dark Matter particles with neutrinos. Because the damping effects are expected to be due to free-streaming neutrinos (i.e. which have already decoupled), we shall estimate the corresponding cross sections at a time $t>t_{\operatorname{dec}(\nu)}$ (where $t_{\operatorname{dec}(\nu)}$ is the neutrino thermal "decoupling" time). Since we are dealing with Dark Matter particles heavier than a few MeV's, the condition $t>t_{d e c(\nu)}$ can be translated in terms of the neutrino energy as: $E_{\nu}<m_{d m}$. Any dependence in $E_{\nu}$ would therefore tend to suppress the cross section. 
$\underline{\text { Scalar Dark Matter particles }}$
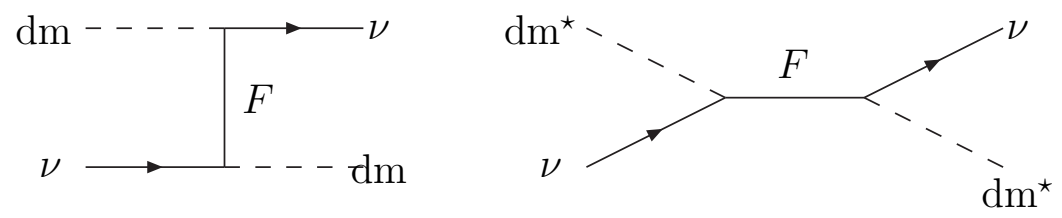

The matrix elements corresponding to the elastic scattering of scalar Dark Matter particles with ordinary neutrinos through a fermion $F$ exchange are given by:

$$
\begin{aligned}
M_{u ; e l} & =i \bar{u}_{\nu_{2}} C_{l} P_{R}\left(p_{\nu_{2}}-p_{d m_{1}}+m_{F}\right) C_{l} P_{L} u_{\nu_{1}} /\left(u-m_{F}^{2}\right), \\
M_{s ; e l} & =i \bar{u}_{\nu_{2}} C_{l} P_{R}\left(p_{\nu_{1}}+p_{d m_{1}}+m_{F}\right) C_{l} P_{L} u_{\nu_{1}} /\left(s-m_{F}^{2}\right) .
\end{aligned}
$$

The $s$-channel does not contribute to the elastic scattering of "non self-conjugate" Dark Matter particles. We make the reasonable assumption of chiral couplings $d m-\bar{\nu}-F^{0}$ (which, within conventions defined in Section 2, means $C_{r}=0$ ). This assumption actually kills the contribution in $m_{F}$ in the numerator, thereby preventing the elastic cross section to be "very" large. From the matrix elements given above, we found:

$$
\begin{aligned}
|M|_{d m \neq d m^{\star} ; e l}^{2} & =\frac{4 C_{l}^{4}}{\left(t-m_{F}^{2}\right)^{2}}\left[\left(p_{d m_{1}} \cdot p_{\nu_{1}}\right)\left(p_{d m_{1}} \cdot p_{\nu_{2}}\right)-\frac{m_{d m}^{2}}{2}\left(p_{\nu_{1}} \cdot p_{\nu_{2}}\right)\right] \\
& =\frac{C_{l}^{4}}{\left(u-m_{F}^{2}\right)^{2}}\left(-s u+m_{d m}^{4}\right)
\end{aligned}
$$

and

$$
|M|_{d m=d m^{\star} ; e l}^{2}=0 \quad \text { in the local limit, }
$$

The reason why $|M|_{d m=d m^{\star} ; e l}^{2}$ vanishes identically in the local limit can be understood by rewriting the two matrix elements $M_{t}, M_{u}$ with the use of the Dirac equation. The latter reduce (in the local limit approximation) to:

$$
\begin{aligned}
M_{u} & =-C_{l}^{2} \bar{u}_{\nu_{2}} p_{d m_{1}} P_{L} u_{\nu_{1}} / m_{F}^{2}, \\
M_{s} & =C_{l}^{2} \bar{u}_{\nu_{2}} p_{d m_{1}} P_{L} u_{\nu_{1}} / m_{F}^{2},
\end{aligned}
$$

so we find indeed $M_{u}+M_{s}=0$. A similar cancellation $\left(M_{u}+M_{s}=0\right)$ also occurs when computing the annihilation process of two self-conjugate scalar Dark Matter particles in a pair neutrino-antineutrino. This can be checked by replacing $m_{f}$ and $C_{r}$ by zero in the results found in Section 9.1.3. On top of that, a partial wave analysis indicates that each of these matrix elements should be proportional to the neutrino energy so that the cross section for 
$d m \neq d m^{\star}$ is indeed expected to be suppressed proportionately to the square of the neutrino energy.

Let us now estimate the contribution of a new gauge boson.

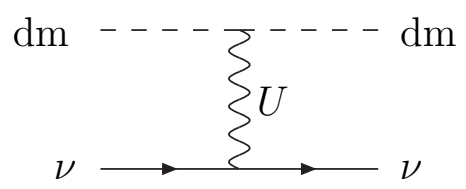

The only possible diagram is a $t$-channel. The corresponding matrix element can be written as:

$$
M_{t}=Q^{\nu} C_{U} f_{U_{l}}\left(-g_{\mu \nu}+\frac{k_{\mu} k_{\nu}}{m_{U}^{2}}\right) \frac{\bar{u}_{\nu_{2}} \gamma^{\mu} P_{L} u_{\nu_{1}}}{\left(t-m_{U}^{2}\right)}
$$

where $Q^{\nu}=\left(p_{d m_{1}}+p_{d m_{2}}\right)^{\nu}$ and $k_{\nu}=\left(-p_{d m_{1}}+p_{d m_{2}}\right)_{\nu}$. This provides:

$$
\left|M_{t}\right|^{2}=\frac{C_{U}^{2} f_{U_{l}}^{2}}{\left(t-m_{U}^{2}\right)^{2}}\left((s-u)^{2}+t\left(4 m_{d m}^{2}-t\right)\right)
$$

which is proportional to the square of the neutrino energy.

$\underline{\text { Fermionic Dark Matter particles }}$
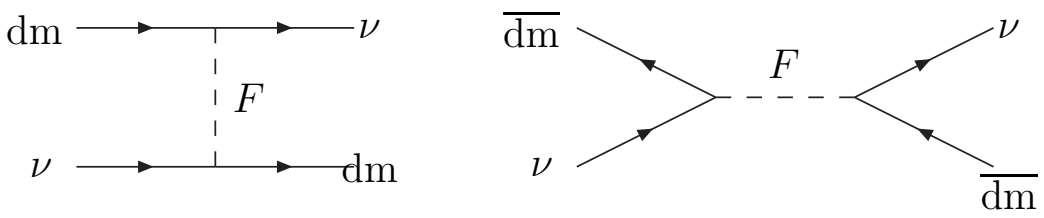

The matrix elements corresponding to the elastic scattering of fermions on neutrinos (disregarding the $U$ contribution) are given by:

$$
\begin{aligned}
& M_{u ; e l}=\bar{u}_{\nu_{2}} C_{l} P_{R} u_{d m_{1}} \frac{1}{u-m_{F}^{2}} \bar{u}_{d m_{2}} C_{l} P_{L} u_{\nu_{1}} \\
& M_{s ; e l}=\bar{u}_{\nu_{2}} C_{l} P_{R} v_{d m_{2}} \frac{1}{s-m_{F}^{2}} \bar{v}_{d m_{1}} C_{l} P_{L} u_{\nu_{1}}
\end{aligned}
$$

Once again, the $s$-channel is expected to only contribute to the cross section associated with Majorana particles (but the $s$-channel actually also contributes for Dirac particles which would interact with antineutrinos). We found: 


$$
\begin{aligned}
|M|_{d m \neq \overline{d m}}^{2} & =4 C_{l}^{4}\left(p_{d m_{1}} \cdot p_{\nu_{2}}\right)\left(p_{d m_{2}} \cdot p_{\nu_{1}}\right) /\left(u-m_{F}^{2}\right)^{2} \\
& =C_{l}^{4} \frac{\left(u-m_{d m}^{2}\right)^{2}}{\left(u-m_{F}^{2}\right)^{2}}
\end{aligned}
$$

and

$$
|M|_{d m=\overline{d m}}^{2}=C_{l}^{4}\left(\frac{\left(u-m_{d m}^{2}\right)^{2}}{\left(u-m_{F}^{2}\right)^{2}}+\frac{\left(s-m_{d m}^{2}\right)^{2}}{\left(s-m_{F}^{2}\right)^{2}}\right) \text {. }
$$

Unlike scalars, there is no cancellation when $d m=\overline{d m}$ so that both Majorana and Dirac candidates are expected to yield a collisional damping effect, whether this effect turns out to be physically relevant or not. Here, we do not estimate the contribution of a new gauge boson (although potentially useful) since fermionic Dark Matter is not the main interest of this paper.

\subsection{Constraints from $g-2$}

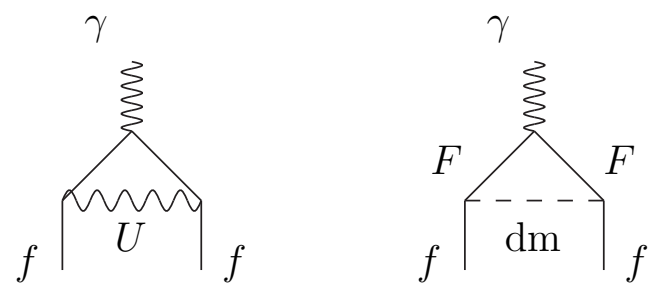

One of the most stringent constraints on the existence of a light gauge boson and light Dark Matter particles coupled to new fermions $F$ comes from the muon and electron anomalous magnetic moments. The corresponding contributions can be derived from [45].

- The diagram associated with the possible existence of a new neutral gauge boson $U$ is very similar to the $Z$ contribution.

When $m_{\mu}>m_{U}>m_{e}$, the contribution of the $U$ boson to the anomalous mag-

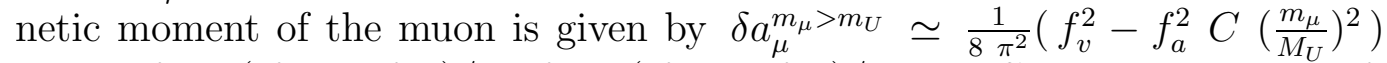
where $f_{v}=\left(f_{U_{l}}+f_{U_{r}}\right) / 2, f_{a}=\left(f_{U_{l}}-f_{U_{r}}\right) / 2$ and $C$ is a numerical coefficient. In order to avoid a potentially large (negative) contribution from $f_{a}$, and also for simplicity, we shall assume $f_{U_{l}}=f_{U_{r}}$, i.e. a vector coupling of the new gauge boson. This may be naturally obtained by using one extra Higgs singlet, in addition to the standard Higgs doublet, to trigger the spontaneous breaking of the $S U(3) \times S U(2) \times U(1) \times U(1)$ gauge group into the $S U(3) \times U(1)$ subgroup of QCD $\times$ QED [16], as discussed in subsection 3.5. 
Table 1

We display the expressions of the coupling $\left(f_{U_{l}}=f_{U_{r}}\right)$ of the $U$ boson to the muon $(U-\mu-\bar{\mu})$ and electron $(U-e-\bar{e})$ in terms of $\delta a_{\mu}$ and $\delta a_{e}$, the extra contributions of the $U$ boson to the muon and electron anomalous magnetic moments. We also give the value of $C_{U}$, the $U$ boson coupling to the Dark Matter, that we obtain by using the relic density condition $i$.e. by imposing that $\left\langle\sigma_{a n n} v_{r e l}\right\rangle_{a n n}$ associated with the virtual production of a $U$ boson be equal to $10^{-27}-10^{-26} \mathrm{~cm}^{3} \mathrm{~s}^{-1}$. $C_{U}$ is then given for both $10^{-27}$ and $10^{-26} \mathrm{~cm}^{3} \mathrm{~s}^{-1}$. We assume $m_{d m} \gtrsim 2 m_{f}, m_{U} \gtrsim 2 m_{d m}$.

\begin{tabular}{|c|c|c|c|c|}
\hline $\begin{array}{c}m_{U} \\
\left(>2 m_{d m}\right)\end{array}$ & $\delta a_{\mu}$ & $\begin{array}{c}U \text { coupled to } \mu: f_{U_{l}} \\
U \text { coupled to } \mathrm{dm}: C_{U}\end{array}$ & $\delta a_{e}$ & $\begin{array}{c}U \text { coupled to } e: f_{U_{l}} \\
U \text { coupled to } \mathrm{dm}: C_{U}\end{array}$ \\
\hline$m_{d m}>m_{\mu}$ & $\simeq \frac{f_{U_{l}}^{2}}{12 \pi^{2}} \frac{m_{\mu}^{2}}{m_{U}^{2}}$ & $\begin{aligned} & f_{U_{l}} \simeq 310^{-6} \\
& \quad\left(\frac{m_{U}}{\mathrm{MeV}}\right)\left(\frac{\delta a_{\mu}}{10^{-9}}\right)^{1 / 2} \\
& C_{U} \simeq(1-4) 10^{-2} \\
& \quad\left(\frac{m_{U}}{m_{d m}}\right)\left(\frac{\delta a_{\mu}}{10^{-9}}\right)^{-1 / 2}\end{aligned}$ & 更 & $\begin{aligned} f_{U_{l}} & \simeq 710^{-5} \\
& \left(\frac{m_{U}}{2}\right)\left(\frac{\delta a_{e}}{s}\right)^{1 / 2}\end{aligned}$ \\
\hline$m_{d m}<m_{\mu}$ & $\simeq \frac{f_{U_{l}}^{2}}{8 \pi^{2}}$ & $\begin{array}{c}f_{U_{l}} \simeq 310^{-4}\left(\frac{\delta a_{\mu}}{10^{-9}}\right)^{1 / 2} \\
\text { [ no annihilation } \\
\text { into } \mu^{+} \mu^{-} \text {possible ] }\end{array}$ & & $\begin{array}{l}C_{U} \simeq(0.5-2) 10^{-3} \\
\left(\frac{m_{U}}{m_{d m}}\right)\left(\frac{\delta a_{e}}{10^{-11}}\right)^{-1 / 2}\end{array}$ \\
\hline
\end{tabular}

This implies $\delta a_{\mu}^{m_{\mu}>m_{U}} \simeq 1.310^{-2} f_{U_{l}}^{2}$, that we have to compare with the difference between the experimental and standard model value, say (1-3) $10^{-9}$ (extendable up to 3 or $510^{-9}$ ) [46]. From this comparison, one gets that $f_{U_{l}}$ or $f_{U_{r}}$, associated with the vector coupling $U-\mu-\bar{\mu}$ should be lower than about $(3-5) 10^{-4}$. Similarly, imposing that the contribution to the anomalous magnetic moment of the electron $\left(\delta a_{e}^{m_{U}>m_{e}} \simeq \frac{f_{U_{l}}^{2}}{12 \pi^{2}} \frac{m_{e}^{2}}{m_{U}^{2}}\right)$ be smaller than a few $10^{-11}$ (up to $810^{-11}$ ), we find that $f_{U_{l}}\left(=f_{U_{r}}\right)$ associated with the coupling $U-e-\bar{e}$ should be smaller than $(7-20) 10^{-5}\left(\frac{m_{U}}{\mathrm{MeV}}\right)$, which gives at most $(7-20) 10^{-3}$ when $m_{\mu}>m_{U}>m_{e}$.

The coupling $C_{U}$ can be obtained by using the relic density argument, once the coupling $f_{U_{l}}$ fixed by the electron $g-2$ (and potentially by the muon $g-2$, if one assumes a universal relationship between the couplings of Dark Matter to the muon and electron). Note that the study of the muon $g-2$ does not allow to derive the value of $C_{U}$ for $m_{\mu}>m_{U}$ since Dark Matter (assume to be lighter than the $U$ boson) cannot annihilate into muons. Finally, for $m_{U} \gtrsim 10$ $\mathrm{MeV}, \delta a_{e}=310^{-12}$, we find $f_{U_{l}}=f_{U_{r}} \lesssim 410^{-4}$ and $C_{U} \simeq 410^{-3}$. One can 
do the same exercise for $m_{U}>m_{\mu}$; the corresponding values can be obtained by using the Table.

- A new (charged) fermionic particle $(F)$ will contribute to the $g-2$ of the muon and electron (hereafter denoted $l$ ) thanks to a diagram which involves two $F$ particles and one Dark Matter particle. Using [45], the contribution of the new $F$ particles is given by

$$
\delta a_{l} \simeq \frac{m_{l}^{2}}{32 \pi^{2}} \int_{0}^{1} d x \frac{\left(C_{l}+C_{r}\right)^{2}\left(x^{2}-x^{3}+x^{2} \frac{m_{F}}{m_{l}}\right)+\left(C_{l}-C_{r}\right)^{2}\left(x^{2}-x^{3}-x^{2} \frac{m_{F}}{m_{l}}\right)}{m_{l}^{2} x^{2}+\left(m_{F}^{2}-m_{l}^{2}\right) x+m_{d m}^{2}(1-x)}
$$

When $m_{F} \gg m_{l}$ and $C_{l} C_{r} \neq 0$ (which is the case we are interesting in) the contribution $\delta a_{l}^{m_{F} \gg m_{l}} \simeq \frac{C_{l} C_{r} m_{l}}{16 \pi^{2} m_{F}}$ is found to be proportional to $m_{l} / m_{F}$ rather than to $\left(m_{l} / m_{F}\right)^{2}$. To satisfy the $g-2$ constraints, the product $C_{l} C_{r}$ should then be about $10^{-3}\left(m_{F} / 100 \mathrm{GeV}\right)$ (or smaller). This is actually in contradiction with the relic density condition which imposes $C_{l} C_{r} \sim 10^{-2}\left(m_{F} / 100 \mathrm{GeV}\right)$.

It therefore seems impossible to satisfy these two conditions simultaneously. However, other particles could bring a similar contribution to $g-2$ but with an opposite sign, thus cancelling the $F$ contribution. This can be achieved, for instance, by introducing a neutral fermion $F^{0}$, a charged scalar $H$ with a mass $m_{F} \sim m_{F^{0}} \gg m_{H} \gg m_{d m}$ and a coupling $F^{0}-H-l$ (somewhat equivalent in supersymmetry to $\left.\chi^{0}-\tilde{l}-l\right)$ of the same order as $F$-dm- $l$. The additional contribution to $g-2$ (associated with the diagram with two $H$ and a $F^{0}$ ) would be given (for $m_{F^{0}} \gg m_{H}$ ) by $a_{l}^{H} \sim-\frac{C_{l H} C_{r} m_{l}}{16 \pi^{2} m_{F^{0}}}=-\frac{C_{l H} C_{r H}}{C_{l} C_{r}} a_{l}^{F}$. Therefore the contribution $a_{l}^{H}$ could actually cancel that of $a_{l}^{F}$ if one requires $C_{l H} C_{r H} \simeq C_{l} C_{r}$ and some fine tuning between the $F$ and $F^{0}$ masses (basically one can use for instance $m_{H} \gtrsim O(100) \mathrm{GeV}, m_{F^{0}} \sim 10 m_{H}$, and $\left.m_{F^{-}} \sim 11.8 m_{H}\right)$. Such a spectrum might lead us to consider a multiplet including a neutral scalar Dark Matter particle together with heavy charged scalar.

Such a cancellation can also occur quite naturally in the framework of theories originating from $N=2$ extended supersymmetry or extra dimensions (cf. Section 8), through the introduction of a new light neutral spin-0 particle. Both Dark Matter and the new neutral spin-0 particle would have non chiral couplings but one would be a scalar and the other one, a pseudoscalar. Their respective contributions to the muon or electron $g-2$ are expected to be of the same magnitude but of opposite sign. Their sum would be therefore naturally small (of the order of $\left(m_{\mu} / m_{F}\right)^{2}$ ), whether each individual contribution is large or not. This actually allows for a sufficiently large value of the annihilation cross section without being in conflict with $g-2$ constraints. 
In a $N=2$ supersymmetric framework for example, both scalar and pseudoscalar spin-0 photons would have, separately, non chiral couplings to muons/ mirror muon (or electron/mirror electrons) pairs. The non chiral character of the couplings of a relatively light scalar spin-0 photon $\left(a_{\gamma}\right)$, here considered as a possible Dark Matter candidate, would allow for sufficiently large annihilation cross sections $\left(a_{\gamma} a_{\gamma} \rightarrow f \bar{f}\right)$. The pseudoscalar spin-0 photon field $\left(b_{\gamma}\right)$ could have a somewhat larger mass (of e.g. a few GeV's, for example). The dominant contributions of the $a_{\gamma}$ and $b_{\gamma}$ exchanges to the charged lepton $g-2$ 's would then largely cancel out. This may be easily understood since the complex combination $\left(a_{\gamma}-i b_{\gamma}\right) / \sqrt{2}$ behaves (as far as $g-2$ is concerned and as long as the mass splitting between the two spin-0 photons is not too large compared to the mirror fermion mass $m_{F}$ ), as a single complex field with chiral couplings, resulting globally in a small (positive) contribution to $a_{\mu}, e \propto \frac{\alpha}{6 \pi} m_{\mu, e}^{2} / m_{F}^{2}$ (while the contribution is $\propto m_{\mu, e} / m_{F}$ for $a_{\gamma}$ and $b_{\gamma}$ separately), which is lower than about $10^{-9}$ for $m_{F} \geq 100 \mathrm{GeV}$.

\section{$9.5 U$ decay}

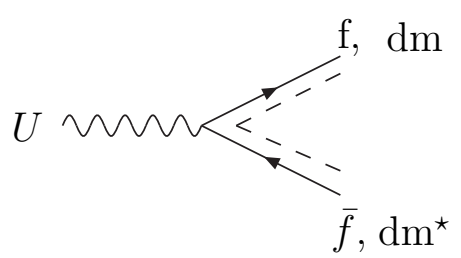

The new gauge boson is supposed to be massive enough to decay into a pair fermion-antifermion (e.g. $e^{+} e^{-}$and of course $\left.\nu \bar{\nu}\right)$, and, if there is enough phase space, into two Dark Matter particles. The two body decay rate into a fermionantifermion pair is given by $\Gamma_{U}^{f} \simeq \frac{m_{U} f_{U l}^{2}}{12 \pi}$, (assuming vectorial couplings $f_{U l}=$ $f_{U r}$ for massive fermions), and the corresponding partial lifetime by

$$
\tau_{U}^{f} \sim 2.510^{-12}\left(m_{U} / \mathrm{MeV}\right)^{-1}\left(f_{U l} / 10^{-4}\right)^{-2} \mathrm{~s} .
$$

The two body decay rate into two spin-0 (non self-conjugate) Dark Matter particles is, on the other hand, given by $\Gamma_{U}^{d m} \simeq \frac{m_{U} C_{U}^{2}}{48 \pi} \simeq \frac{\Gamma_{U}^{f} C_{U}^{2}}{\left(4 f_{U l}^{2}\right)}$, which implies

$$
\tau_{U}^{d m} \sim 10^{-11}\left(m_{U} / \mathrm{MeV}\right)^{-1}\left(C_{U} / 10^{-4}\right)^{-2} \mathrm{~s}
$$

With $C_{U}=310^{-3}$, as obtained if $\delta a_{e}=310^{-12}, m_{d m}=4 \mathrm{MeV}$ and $m_{U} / m_{d m}=2.5$, one gets $\tau_{U}^{d m} \sim 10^{-15} \mathrm{~s}$. The corresponding value of $f_{U_{l}}$ for these parameters being of $410^{-4}$, we find $\tau_{U}^{e} \sim 1.510^{-14} \mathrm{~s}$. In such situations the decay into two Dark Matter particles can dominate over the decay into a pair fermion-antifermion (with a branching ratio $B_{f}=\Gamma_{f} / \Gamma_{t o t}$ of about $610^{-2}$ ). The $U$ boson would then mainly decay into "missing energy" and 
would, most likely, also escape searches in nuclear transitions [47]. This therefore indicates that, for some values of the parameters, the coupling of the $U$ boson with Dark Matter can allow for a short $U$ boson lifetime without violating the $g-2$ constraints (i.e. without requiring a cancellation mechanism so as to satisfy the $g-2$ constraints)!

\subsection{Constraints from direct $U$ production and initial state radiation}

The existence of a $U$ boson could also be constrained, directly or indirectly, by accelerator searches, through the processes $e^{+} e^{-} \rightarrow \gamma U$ or $e^{+} e^{-} \rightarrow U \rightarrow$ $\mathrm{dm} \mathrm{dm}$. Once produced directly (i.e. $e^{+} e^{-} \rightarrow \gamma U$ ), the $U$ boson may have invisible and visible decay modes (depending on whether it decays into $\mathrm{dm} \mathrm{dm}$, $\nu \bar{\nu}$ or $e^{+} e^{-}$, for instance). The invisible modes are expected to be dominant, according to the branching ratios discussed previously, but the visible modes could nevertheless be important depending on the cross section associated with the $U$ boson production.

\section{Direct $U$ boson production}
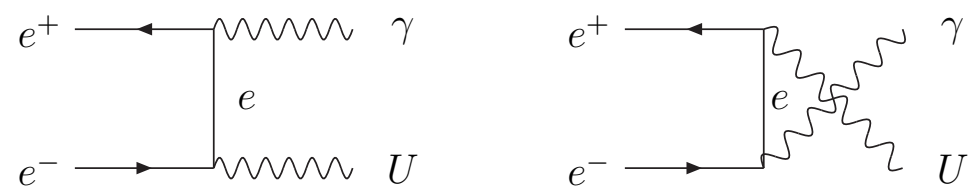

This process is similar to $e^{+} e^{-}$annihilation into two photons (especially for $\sqrt{s}>10 \mathrm{MeV}$ ) in which one would replace a photon by a $U$ boson. One therefore expects the associated differential cross section $\left(\frac{d \sigma}{d \Omega}\right)_{U \gamma}$ to be, at energy $E_{e}>m_{U}$, equal to $\left(\frac{d \sigma}{d \Omega}\right)_{U \gamma} \simeq 2\left(f_{U_{l}}^{2} / e^{2}\right)\left(\frac{d \sigma}{d \Omega}\right)_{\gamma \gamma}$, say $\frac{e^{2} f_{U_{l}}^{2}}{s} \frac{1+\cos \theta}{\sin ^{2} \theta}$, in the centre-of-mass frame (with $s=4 E_{e}^{2}$ ). The $U$ boson's production should therefore be suppressed by a factor $\lesssim 10^{-4}$ as compared to the annihilation into two photons. With the value $f_{U_{l}} \sim 710^{-5} \frac{m_{U}}{\mathrm{MeV}}\left(\frac{\delta a_{e}}{10^{-11}}\right)^{1 / 2}$ as mentioned earlier, we get a suppression factor of about $510^{-8}\left(\frac{m_{U}}{\mathrm{MeV}}\right)^{2}\left(\frac{\delta a_{e}}{10^{-11}}\right)$. This implies a cross section smaller than

$$
\left(\frac{d \sigma}{d \Omega}\right)_{U \gamma} \simeq 510^{-37}\left(\frac{E_{e}}{1 \mathrm{GeV}}\right)^{-2}\left(\frac{f_{u_{l}}}{410^{-4}}\right)^{2} \frac{1+\cos \theta}{\sin ^{2} \theta} \mathrm{cm}^{2}
$$

which seems large enough to be of interest for collider experiments depending on $f_{U_{l}}$. However, even in the case of a large cross section, very energetic photons, as produced in this process, might be easily confused with that orig- 
inating from QED events (especially if the $U$ then decays in visible modes) so it might be difficult to exclude such a possibility.

If the $U$ boson mainly decays into Dark Matter, then the $U$ production process turns out to be of the type $e^{+} e^{-} \rightarrow \gamma+\not B$, where $\not$ is missing energy, which is of interest in experiments searching for single photon production events. But, in the case of a light Dark Matter candidate, such a process is likely to remain unobserved, owing to the large background associated with $e^{+} e^{-} \rightarrow \gamma \gamma$, in which one of the two photons escapes detection.

Still it may be useful to compare our estimate to the sensitivity of "neutrino counting" experiments, where two neutrinos are produced in $e^{+} e^{-}$annihilations and a single photon is used to trigger on the event $\left(e^{+} e^{-} \rightarrow \nu \bar{\nu} \gamma\right)$. The standard model cross section, at $\sqrt{s}=29 \mathrm{GeV}$, for a tag photon with an energy greater than $1 \mathrm{GeV}$ in the direction $\theta_{\gamma}>20^{\circ}$, is about $0.04 \mathrm{pb}$ for $N_{\nu}=3$ (and greater for $N_{\nu}>3$ ). The experimental constraint, at this energy, is $N_{\nu}<7.9$ at $90 \% \mathrm{CL}$ [48], which corresponds to a cross section of $0.07 \mathrm{pb}$. Therefore extra particles with a cross section lower than 0.03 pb at $\sqrt{s}=29 \mathrm{GeV}$ are, in principle, allowed. At LEP, where the limit on $N_{\nu}$ is much better because the energy in the centre-of-mass frame is closer to the $Z$ pole $[49,50]$, we expect the process $e^{+} e^{-} \rightarrow U \gamma$ to be of the order of $\sigma \sim 510^{-41}\left(\frac{f_{u_{l}}}{410^{-4}}\right)^{2} \mathrm{~cm}^{2}$, say much lower than the cross section $e^{+} e^{-} \rightarrow \nu \bar{\nu} \gamma$. This again indicates that the existence of a $U$ boson should have escaped searches for "missing energy".

\section{Anomalous single photon production}

Here are the diagrams involved in Initial State Radiation (ISR) mechanism:
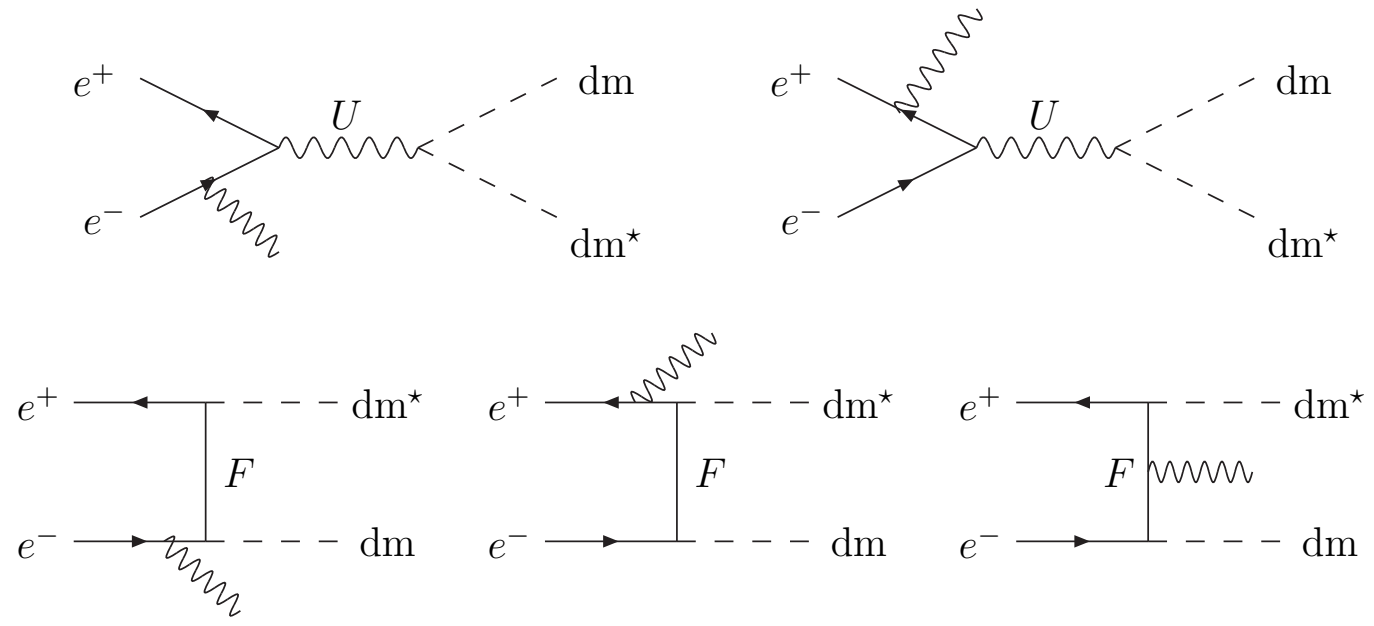

Because the direct production of Dark Matter particles from $e^{+} e^{-}$annihilations consists only in "missing energy" (carried away by the two unobserved Dark Matter particles), a possible signature to search for in accelerator experi- 
ments consists in the emission, by the incoming particles (or by the exchanged particles when one deals with a charged $F$ ), of a single photon:

$$
e^{+} e^{-} \longrightarrow \gamma \mathrm{dm} \mathrm{dm} \text {. }
$$

This process is similar to the one involved in neutrino, neutralino or sneutrino searches so we can use existing limits to determine whether light Dark Matter particles can be viable or not.

The diagrams are basically the same as for sneutrino production but the $Z$ boson is replaced by a $U$ boson and the chargino by a $F$ particle [48]. The annihilations through the virtual production of a $U$ and a $Z$ boson seem at a first glance similar (the smallness of the couplings compensates the difference of mass between the $Z$ and $U$ bosons). However, because we are considering a light gauge boson, the cross section - usually proportional to $G_{F}^{2} E^{2} / 12 \pi \sim 10^{-39}(E / \mathrm{GeV})^{2} \mathrm{~cm}^{2}$ for $E<M_{Z}$ for ordinary weak interactions - is replaced by $f_{U_{l}}^{2} C_{U}^{2} / E^{2} \lesssim 610^{-42}\left(\frac{m_{U}}{\mathrm{MeV}}\right)^{4}\left(\frac{m_{d m}}{\mathrm{MeV}}\right)^{-2}(E / \mathrm{GeV})^{-2} \mathrm{~cm}^{2}$. This indicates that the pair production of Dark Matter particles through the virtual production of a $U$-boson is indeed generally lower than the weakinteraction production of neutrino pairs. Anomalous single photon production due to $e^{+} e^{-} \rightarrow \operatorname{dm} \operatorname{dm} \gamma$ would then have escaped even "low energy" experiments (in particular, those appropriate for neutrino counting like PETRA and PEP experiments), if they proceed through the virtual production of a $U$ boson.

The Dark Matter production through $F$ exchanges seems, on the other hand, much closer to experimental limits, and therefore more interesting for accelerator experiments. The matrix elements associated with the emission of a photon by the incoming particles are given by:

$$
\begin{aligned}
& M_{1}=\frac{e}{D_{1}} \overline{v_{1}}\left(C_{l} P_{R}+C_{r} P_{L}\right)\left(p \not k+m_{F}\right)\left(C_{l} P_{L}+C_{r} P_{R}\right)\left(p \not 2-\not k+m_{e}\right) k u_{2}, \\
& M_{2}=\frac{e}{D_{2}} \overline{v_{1}} k\left(p h-\not k+m_{e}\right)\left(C_{l} P_{R}+C_{r} P_{L}\right)\left(p \not t+m_{F}\right)\left(C_{l} P_{L}+C_{r} P_{R}\right) u_{2},
\end{aligned}
$$

while the matrix amplitude associated with the emission of a photon by the exchanged particle can be written as:

$$
M_{3}=\frac{e}{D_{3}} \overline{v_{1}}\left(C_{l} P_{R}+C_{r} P_{L}\right)\left(p_{F / 1}+m_{F}\right) k\left(p_{F / 2}+m_{F}\right)\left(C_{l} P_{L}+C_{r} P_{R}\right) u_{2},
$$

with $p_{F_{1}}=p_{e_{1}}-p_{d m_{1}}, p_{F_{2}}=p_{e_{2}}-p_{d m_{2}}, D_{1}=2 k \cdot p_{2}\left(p_{F}^{2}-m_{F}^{2}\right), \quad D_{2}=$ $2 k \cdot p_{1}\left(p_{F}^{2}-m_{F}^{2}\right)$ and $D_{3}=\left(\left(p_{e_{1}}-p_{d m_{1}}\right)^{2}-m_{F}^{2}\right)\left(\left(p_{e_{2}}-p_{d m_{2}}\right)^{2}-m_{F}^{2}\right)$. The leading term in each squared matrix amplitude associated with (13) is given, in the 
case of chiral couplings and neglecting the electron mass, by $8\left(C_{l} C_{r}\right)^{2} / m_{F}{ }^{2}$. The leading term in the squared matrix amplitude associated with (14) is, on the other hand, given by $4 E_{e}^{2}\left(C_{l}^{4}+C_{r}^{4}\right) / m_{F}^{4}$ for $E_{e}<m_{F}$. Since we consider values of $m_{F}$ greater than $100 \mathrm{GeV}$, the squared matrix amplitude associated with (14) appears slightly suppressed compared to that associated with (13). We can therefore neglect (14) to get a first estimate.

Owing to the relation $C_{l}^{2} C_{r}^{2} / m_{F}^{2} \approx 4 \pi \sigma_{a n n} v_{r e l}$ (where we impose $\sigma_{a n n} v_{\text {rel }} \gtrsim$ $10^{-26}-10^{-27} \mathrm{~cm}^{3} \mathrm{~s}^{-1}$ so as to justify an asymmetry, as necessary if $m_{d m} \lesssim 100$ $\mathrm{MeV})$, the single photon production ${ }^{18}$ cross section $e^{+} e^{-} \rightarrow \operatorname{dm} \operatorname{dm} \gamma$ is expected to be of the order of $\sigma^{\gamma} \sim 4 \alpha x\left\langle\sigma_{\text {ann }} v_{\text {rel }}\right\rangle / 4 \pi$ c, i.e. $\sigma^{\gamma} \approx$ $x \frac{\left\langle\sigma_{a n n} v_{r e l}\right\rangle}{10^{-26} \mathrm{~cm}^{3} \mathrm{~s}^{-1}} \mathrm{fb},\left(x\right.$ being defined as $E_{\gamma}=x E_{e}$ and assuming $E_{e} \gg m_{d m}>$ $m_{e}$ ). This order of magnitude (although close enough to experimental limits to justify further studies since $\sigma^{\gamma} \sim \mathcal{L}_{\text {LEP }}^{-1}$ ), tends to suggest that light Dark Matter particles could have indeed escaped previous collider (e.g. PEP, LEP) experiments. (We note moreover that $\sigma^{\gamma}$ is also very close to the (LSP) neutralino-associated single-photon process for neutralino masses larger than present experimental limits.)

U-strahlung.
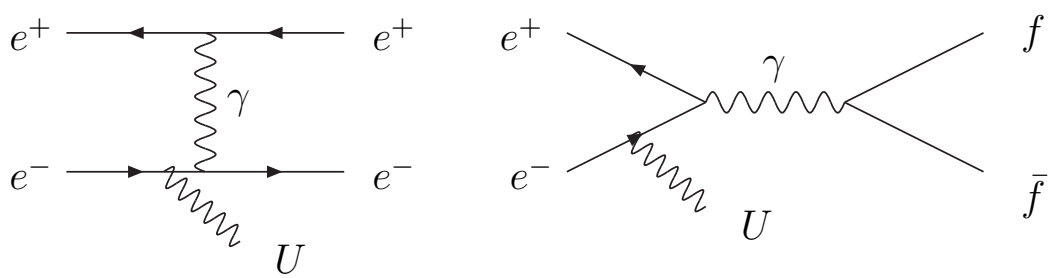

These are examples of $U$ strahlung. Because of the smallness of the $U$ boson coupling to ordinary particles, such processes are expected to be at least $310^{-7}\left(m_{U} / \mathrm{MeV}\right)^{2}$ times lower than the single photon production process $\left(e^{+} e^{-} \rightarrow f \bar{f} \gamma\right)$. These should be difficult to detect for a light $U$ boson having a mass $m_{d m}<O(\mathrm{GeV})$.

\section{References}

[1] J. Silk, Ap. J. 151, 459 (1968).

[2] The Macho collaboration, Ap. J. Lett. 550, L169 (2001).

[3] M. Davis, M. Lecar, C. Prior, E. Witten, Ap. J. 250, 423 (1981).

${ }^{18}$ The cross section actually also depends on a term inversely proportional to $x$ but it is weighted by the square of the electron mass and is therefore suppressed. 
[4] J. E. Gunn et al., Ap. J. 223, 1015G (1978); P. J. E. Peebles, Ap. J. 263L, 1P (1982).

[5] B. Moore, Nature, 370, 629 (1974); R.A. Flores, J.R. Primack, Ap. J. Lett. 457, L5 (1994); A. Burkert, Ap. J. Lett. 447, L25 (1995).

[6] F.C. van den Bosch, R. A. Swaters, accepted for publication MNRAS, astro$\mathrm{ph} / 0006048$.

[7] W.J.G. De Blok, A. Bosma, S.S. McGaugh, accepted for publication MNRAS, astro-ph/0212102.

[8] The DELPHI Collaboration, P. Abreu et al., Phys. Lett. B489, 38 (2000).

[9] J. Ellis, T. Falk, K.A. Olive, M. Srednicki, Astropart. Phys. 13, 181 (2000); M. E. Gomez, G. Lazarides, C. Pallis, Phys. Rev. D61, 123512 (2000); C. Boehm, A. Djouadi, M. Drees, Phys. Rev. D62, 035012 (2000).

[10] B. Lee, S. Weinberg, Phys. Rev. Lett. 39, 165 (1977).

[11] V. Silveira, A. Zee, Phys. Lett. B161, 136 (1985); D. Holz, A. Zee, Phys. Lett. B517, 239 (2001); M.C. Bento, O. Bertolami, R. Rosenfeld, Phys. Lett. B518, 276 (2001); M.C. Bento, O. Bertolami, R. Rosenfeld, L. Teodoro, Phys. Rev. D62, 041302 (2000). See also J. McDonald, Phys. Rev. Lett. 88, 091304 (2002).

[12] C. Boehm, T. Ensslin, J. Silk, astro-ph/0208458.

[13] P. Fayet, Phys. Lett. B69, 489 (1977); G. Farrar, P. Fayet, Phys. Lett. B76, 575 (1978).

[14] P. Fayet, Phys. Lett. B95, 285 (1980).

[15] P. Fayet, Nucl. Phys. B187, 184 (1981).

[16] P. Fayet, Phys. Lett. B227, 127 (1989).

[17] P. Fayet, Nucl. Phys. B90, 104 (1975).

[18] P. Fayet, Nucl. Phys. B347, 743 (1990); Class. Quant. Grav. 13, A19 (1996).

[19] R. C. Allen et al., Phys. Rev. D47, 11 (1993); LSND coll., Phys. Rev. D63, 112001 (2001).

[20] P. Fayet, Phys. Lett. B70, 461 (1977).

[21] P. Fayet, M. Mézard, Phys. Lett. B104, 226 (1981).

[22] P. Fayet, Phys. Lett. B171, 261 (1986); Phys. Lett. B172, 363 (1986).

[23] ALEPH, DELPHI, L3 and OPAL Collaborations, Phys. Lett. B565, 61 (2003).

[24] J. S. Hagelin, G. L. Kane, S. Raby, Nucl. Phys. B241, 638 (1984).

[25] see for instance D. N. Spergel et al., astro-ph/0302209; A.H. Jaffe et al., Phys. Rev. Lett. 86, 3475 (2001). 
[26] O. Lahav et al., "New Trends in Theoretical and Observational Cosmology", The 5th RESCEU Symposium, Tokyo, to be published by Universal Academy Press, astro-ph/0205382.

[27] See for instance P. Gondolo et al., Proceedings of the 4th International Workshop on Identification of Dark Matter (idm2002), York, England, 2-6 September, 2002 (astro-ph/0211238).

[28] K. Griest, Phys. Rev. D38, 2357 (1988), Erratum-ibid. D39, 3802 (1989); M. Drees, M. M. Nojiri, Phys. Rev. D47, 376 (1993).

[29] G. Hasinger, A\&A Supp. 120, 607 (1996), and references therein; P. Sreekumar et al., Ap. J. 494, 523 (1998).

[30] J. F. Navarro, C. S. Frenk, S. D.M. White, Ap. J. 462, 563 (1996).

[31] P. McDonald, R. Scherrer, T. Walker, Phys. Rev. D63, 023001 (2001).

[32] C.L. Bennett et al., astro-ph/0302207.

[33] Many authors have done the corresponding calculations. See for instance: H. Goldberg, Phys. Rev. Lett. 50, 19 (1983); J. Ellis et al., Nucl. Phys. B238, 453 (1984); K. Griest, Phys. Rev. D38, 8 (1988) and [28].

[34] C. Boehm, P. Fayet, R. Schaeffer, Phys. Lett. B518, 8 (2001); Proc. "DARK 2002, 4th Int. Conf. on Dark Matter in Astro and Particle Physics", Cape Town (Springer, 2002), p. 333, astro-ph/0205406; and paper in preparation.

[35] C. Boehm, A. Riazuelo, S. H. Hansen, R. Schaeffer, Phys. Rev. D66, 083505 (2002).

[36] V. K. Narayanan et al., Ap. J. Lett. 543, 103N (2000).

[37] N. Yoshida et al., astro-ph/0303622.

[38] A. Knebe et al., astro-ph/0302443.

[39] C. Boehm et al., astro-ph/0309652.

[40] J. Ellis, T. Falk, K. A. Olive, Phys. Lett. B444, 367 (1998); T. Nihei, L. Roszkowski, R. Ruiz de Austri, JHEP 0203, 031 (2002).

[41] P. Fayet, Nucl. Phys. B149, 137 (1979); Phys. Lett. B142, 263 (1984); Nucl. Phys. B246, 89 (1984).

[42] P. Fayet, Phys. Lett. B159, 121 (1985).

[43] B. Delamotte, F. Delduc, P. Fayet, Phys. Lett. B176, 409 (1986); B. Delamotte, P. Fayet, Phys. Lett. B195, 563 (1987).

[44] P. Fayet, Nucl. Phys. B263, 649 (1986); Proc. 2nd Nobel Symp. on El. Part. Physics (Marstrand, Sweden, 1986), Physica Scripta T 15 (1987) 46.

[45] J. P. Leveille, Nucl. Phys. B137, 63 (1978). 
[46] M. Davier et al., Eur. Phys.J. C27, 497 (2003).

[47] F.W.N. De Boer, R. Van Dantzig, Phys. Rev. Lett. 61, 1274 (1988); A. Bross et al., Phys. Rev. Lett. 67, 2942 (1991).

[48] C. Hearty et al., Phys. Rev. D39, 3207 (1989).

[49] The LEP Collaborations: ALEPH Collaboration, DELPHI Collaboration, L3 Collaboration, OPAL Collaboration, the LEP Electroweak Working Group, the SLD Heavy Flavour Working Group, hep-ex/0212036.

[50] L3 Collab., M. Acciarri et al., Phys. Lett. B431, 199 (1998). 